\title{
Electrical contacts to two-dimensional semiconductors
}

\author{
Adrien Allain', Jiahao Kang², Kaustav Banerjee ${ }^{2 \star}$ and Andras Kis ${ }^{1 \star}$
}

The performance of electronic and optoelectronic devices based on two-dimensional layered crystals, including graphene, semiconductors of the transition metal dichalcogenide family such as molybdenum disulphide $\left(\right.$ MoS $\left._{2}\right)$ and tungsten diselenide $\left(\mathrm{WSe}_{2}\right)$, as well as other emerging two-dimensional semiconductors such as atomically thin black phosphorus, is significantly affected by the electrical contacts that connect these materials with external circuitry. Here, we present a comprehensive treatment of the physics of such interfaces at the contact region and discuss recent progress towards realizing optimal contacts for two-dimensional materials. We also discuss the requirements that must be fulfilled to realize efficient spin injection in transition metal dichalcogenides.

S emiconducting electronic devices utilize fine control over the flow of charge carriers, which are injected into the semiconducting material through electrical contacts. The quality of the electrical contacts - quantified through contact resistance - is as important to the proper functioning of the entire device as the semiconductor (SC) itself. Since the early 1990s, researchers have explored a wide variety of electronic devices based on nanostructures with different dimensionalities, ranging from one-dimensional (1D) carbon nanotubes ${ }^{1}, \mathrm{SC}$ nanowires ${ }^{2}$ or $2 \mathrm{D}$ materials, starting with graphene ${ }^{3}$.

Advances in the realization of electronic devices ${ }^{4-6}$, including the recent demonstration of a super-steep-slope atomically thin channel interband tunnelling transistor ${ }^{7}$, optical characterization ${ }^{8,9}$ and material preparation ${ }^{10}$ have revived scientific interest in the single- and few-layer form of layered transition metal dichalcogenides (TMDCs), a material family that has a wide range of electrical properties. This class of materials has a common chemical formula $\mathrm{MX}_{2}$, where $\mathrm{M}$ stands for a transition metal (most commonly Mo, W, $\mathrm{Nb}, \mathrm{Ta}$ or $\mathrm{Ti}$ ) and $\mathrm{X}$ is a chalcogen atom ( $\mathrm{S}$, Se or $\mathrm{Te}$ ). Compounds based on Mo and $\mathrm{W}$ are the best-known examples of semiconducting TMDCs. In their single- and few-layer form they show many attractive features such as atomic-scale thickness and large bandgaps (1-2 eV), leading to a high degree of electrostatic control ${ }^{11}$ and scalability for nanoscale transistors ${ }^{12}$, exquisite sensing capabilities $^{13}$, high breakdown voltages ${ }^{14}$, tunable optical properties ${ }^{8,9,15-17}$, a high degree of mechanical flexibility ${ }^{18}$ and the possibility of engineering new materials through the realization of van der Waals heterostructures ${ }^{19}$. Other semiconducting $2 \mathrm{D}$ materials such as phosphoren $\mathrm{e}^{20}$ and silicene $\mathrm{e}^{21}$ are also attracting growing interest.

One of the most common electronic devices, in both the research and industrial environments, is the field-effect transistor (FET). Low contact resistance in 2D SC-based devices is critical for achieving high 'on' current, large photoresponse ${ }^{22}$ and high-frequency operation $^{23}$. However, the major issue for 2D SC FET transistors is the existence of a large contact resistance at the interface between the 2D SC and any bulk (or 3D) metal, which drastically restrains the drain current ${ }^{24-26}$. Contacting 2D SCs presents a certain number of experimental and conceptual challenges. The theoretical concepts that underlie our understanding of conventional metal-SC contacts break down in the limit where the SC thickness is smaller than the depletion and transfer lengths. In the $2 \mathrm{D}$ limit, the properties of the interface - the chemical interaction between the metal and the SC - govern everything. Substitutional doping, a common strategy adopted to decrease the contact resistance in bulk SCs, is not applicable here because it would modify both the $2 \mathrm{D}$ material and its properties. In addition, the pristine surface (that is, no dangling bonds) of a $2 \mathrm{D}$ material makes it difficult to form strong interface bonds with a metal, thereby increasing contact resistance.

The quantum limit to the contact resistance $\left(R_{\mathrm{C}}^{\min }\right)$ is determined by the number of conducting modes within the SC channel ${ }^{27,28}$, which is connected to the $2 \mathrm{D}$ charge carrier density $\left(n_{2 \mathrm{D}}\right)$, yielding $R_{\mathrm{C}}^{\min }=h /\left(2 e^{2} k_{\mathrm{F}}\right)=0.026 / \sqrt{ } n_{2 \mathrm{D}} \approx 30 \Omega \mu \mathrm{m}$ at $n_{2 \mathrm{D}}=10^{13} \mathrm{~cm}^{-2}$ (ref. 29) a value three orders of magnitude below the typical contact resistance to monolayer $\mathrm{MoS}_{2}$. Here, $h$ is Planck's constant, $k_{\mathrm{F}}$ is the Fermi wavevector and $e$ is the electron charge. There is plenty of room for improvement in this respect, making it all the more important to study the detailed physics of contacts between metals and 2D SCs.

In this Review, we first discuss the geometry and nature of the interfaces between 2D materials and metal contacts. We then describe the charge-injection mechanisms that occur at the contacts and summarize what is known about 'Schottky barriers' to 2D SCs. We then discuss contact resistance and how it scales, and present an overview of current research in this area. Finally, we discuss the influence of contact resistance on spin injection into 2D SCs. Most research into devices based on 2D SCs is carried out using $\mathrm{MoS}_{2}$, owing to its wide availability. Concepts and limitations that we outline here can, however, be readily extended to other 2D SCs.

\section{Interface geometry}

There are two fundamental interface geometries (topologies) between bulk (3D) metals and 2D materials: top contact (Fig. 1a) and edge contact (Fig. 1b), which perform differently.

Pure top contacts can be made by simply avoiding contact between the metal and the edges of the $2 \mathrm{D}$ material ${ }^{30}$. However, the formation of a pure edge contact using standard lithographic techniques is difficult in a single- or few-layer 2D material, owing to its atomically thin body; so far, only one example has been reported ${ }^{31}$ of a purely edge-contacted graphene monolayer. In most experiments, contacts to $2 \mathrm{D}$ materials are a combination of these two geometries. 
a

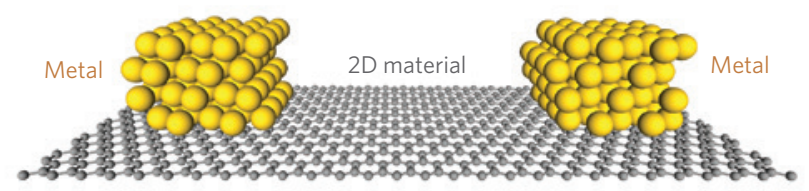

b

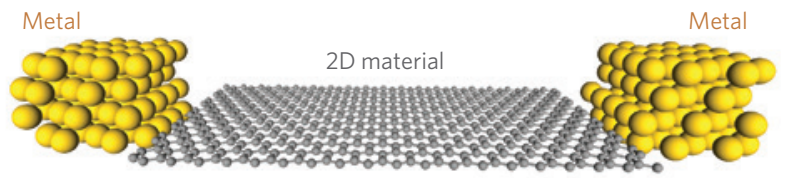

Figure 1 | Interface geometries of metal-2D contacts. a, Top-contact configuration. b, Edge-contact configuration.

Contrary to the case of bulk (3D) SCs (Fig. 2a,b), the pristine surfaces of $2 \mathrm{D}$ materials do not tend to form covalent bonds. The interfaces between metals and 2D materials in the top-contacted configuration can therefore only be formed by a van der Waals (vdW) gap in most situations (Fig. 2c). As shown in Fig. 2d, the vdW gap in such top-contact interfaces acts as an additional 'tunnel barrier' for carriers, before the inherent Schottky barrier (SB) ${ }^{32}$. The tunnel barrier greatly reduces the charge injection from metals, which results in higher contact resistance.

One option to overcome this vdW gap is to take advantage of edge contacts. In fact, edge contacts to monolayer graphene have been modelled ${ }^{33}$ and shown ${ }^{31}$ to perform better than top contacts.
Density functional theory (DFT) has shown that edge contacts lead to shorter bonding distance with stronger hybridization (orbital overlap) than top contacts, and transport simulations show that the incorporation of additional interfacial species (such as oxygen) can help to improve bonding and increase transmission ${ }^{31}$. The reported contact resistance for a $\mathrm{Cr}$ edge contact with monolayer graphene is about $150 \Omega \mu \mathrm{m}$, which is in good agreement with the value of $118 \Omega \mu \mathrm{m}$ predicted from simulations ${ }^{31}$. For TMDC SCs, edge contacts can also be advantageous compared with top contacts. This has been verified by DFT simulations for both monolayer ${ }^{32,34}$ and multilayer TMDCs ${ }^{35}$. The main reasons are the stronger orbital overlaps and the reduction of tunnel barriers.

Edge contacts are particularly relevant to multilayered 2D materials ${ }^{36}$ owing to the large conductivity anisotropy of $2 \mathrm{D}$ materials between the in- and out-of-plane directions. A model accounting for both top and edge contacts to multilayer graphene ${ }^{37}$ showed that, in this case, edge contacts significantly reduce the overall contact resistance ${ }^{36}$. Another model, based on a resistor network and considering the back-gate screening effect ${ }^{38}$, has been used to compare top- and edge-contacted multilayer graphene devices. Using this model, the extracted edge contact resistance from experiments is $150-360 \Omega \mu \mathrm{m}$ for each graphene layer, which is relatively small compared with the tunnelling resistances between each layer ${ }^{38}$. Similar work has been reported ${ }^{39}$ in which the currents flowing through the graphene surface and edges were theoretically and experimentally investigated by patterning graphene under the contact metal with different perimeter-to-area ratios.

Nevertheless, as mentioned previously, most practical contact structures involve both the edge and the top surface of the $2 \mathrm{D}$ a

Metal-bulk SC contact
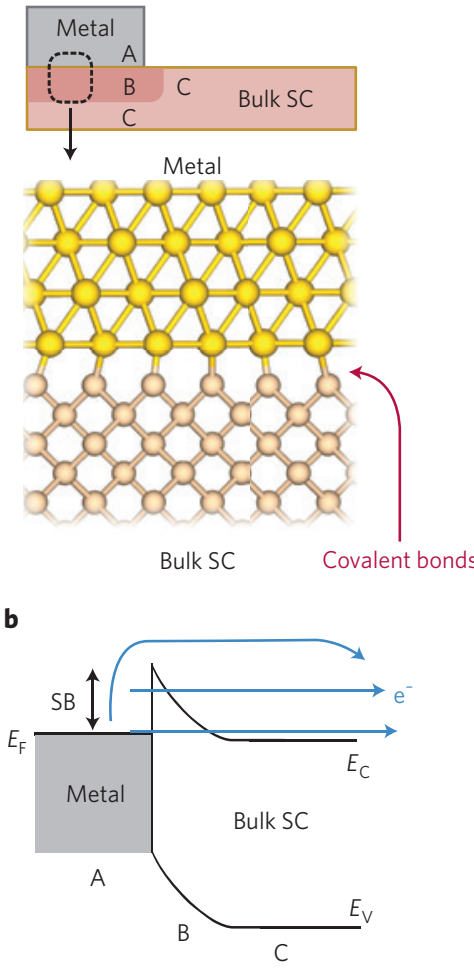

c Metal-2D SC contact (with vdW gap)

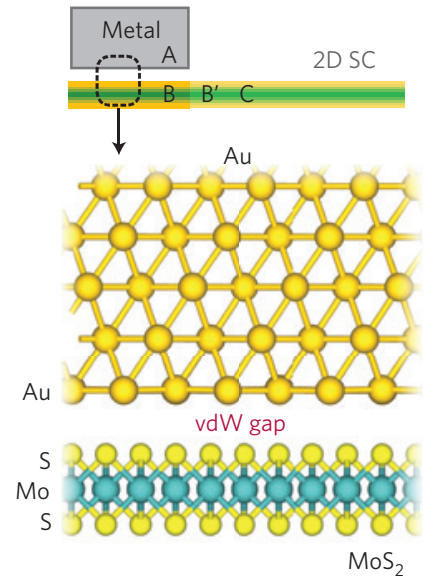

d

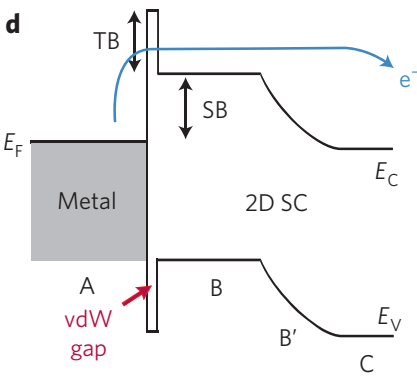

e Metal-2D SC contact (with hybridization)

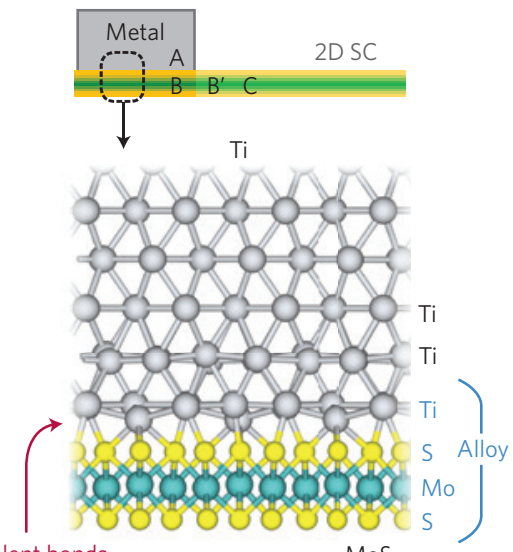

Covalent bonds

$\mathrm{MoS}_{2}$

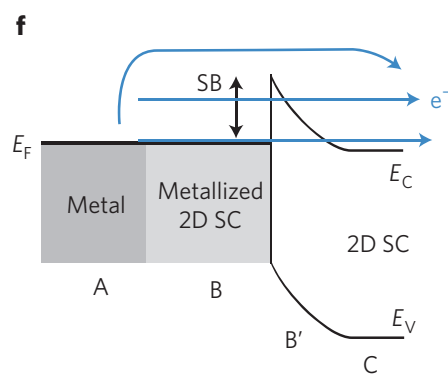

Figure 2 | Different types of metal-SC junction and their respective band diagrams. a,b, Schematic (a) and corresponding band diagram (b) of a typical

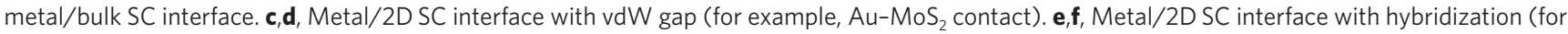
example, Ti-MoS 2 contact, where $\mathrm{MoS}_{2}$ under the contact is metallized by Ti). $E_{\mathrm{F}}, E_{\mathrm{C}}$ and $E_{\mathrm{V}}$ represent the Fermi level of the metal, and the conduction and

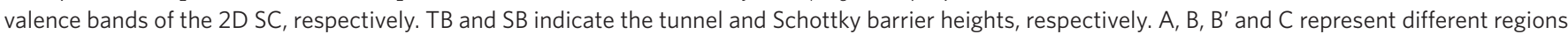
in the current path from the metal to the SC. The blue arrows in $\mathbf{b}$, $\mathbf{d}$ and $\mathbf{f}$ represent the different injection mechanisms. From top to bottom: thermionic emission, thermionic field emission and field emission (tunnelling). In d, only thermionic emission is available. 
material, with the top surface having a greater contribution due to the large surface-area-to-edge-area ratio of the contact. Hence, other than optimizing the edge contacts, the contact resistance can be improved by reducing the tunnel barrier at the top surface. This can be achieved by hybridization between atoms of contact metals and 2D SC surfaces. DFT simulations show that specific metals can form covalent bonds to 2D SC surfaces (Fig. 2e), and hence eliminate the vdW gap; for example, Ni for graphene ${ }^{40}$, Ti for $\mathrm{MoS}_{2}$ (refs 34,41), Pd for $\mathrm{WSe}_{2}$ (ref. 34), Mo/W for $\mathrm{MoS}_{2} / \mathrm{WSe}_{2}$ (refs 32,42) and $\mathrm{Ti}_{2} \mathrm{C}$ (a $2 \mathrm{D}$ metallic material) for $\mathrm{MoS}_{2}$ (ref. 43).

Strong hybridization can also distort the properties of $2 \mathrm{D}$ SCs below the top contacts (especially for a monolayer) ${ }^{42}$. This can modify the sheet resistance of 2D SCs below the contacts $\left(\rho_{\text {contact }}^{2 \mathrm{D}}\right)$, thus causing a change in contact resistance (the calculation of which will be discussed in a later section). It should be noted that hybridization can both increase and decrease $\rho_{\text {contact }}^{2 \mathrm{D}}$. DFT predicts that selected metals such as Ti and Mo create nonlocalized overlap states in the original bandgap of $\mathrm{MoS}_{2}$ (ref. 32), which effectively turns $\mathrm{MoS}_{2}$ under the contact into a new metallic compound (Fig. 2f). In such a situation, $\rho_{\text {contact }}^{2 \mathrm{D}}$ reduces. On the other hand, if the monolayer is partially metallized, $\rho_{\text {contact }}^{2 \mathrm{D}}$ may increase owing to localized states.

It is worth noting that the DFT predictions for cases of strong hybridization (Fig. 2f) are based on the assumption of perfect interfaces. In practice, close-to-perfect interfaces require the removal or prevention of surface impurities (such as resist residues), as well as an annealing process. For example, in graphene, during annealing, the carbon atoms can dissolve into the contact metal (Ni or Co) and thus form strong covalent bonds, which contribute towards a much smaller contact resistance ${ }^{44}$.

For multilayer TMDC SCs, only the top layer can be hybridized by metal top contacts, and thus only the vdW gap between the metal and the top layer of the TMDC is eliminated, as predicted by $\mathrm{DFT}^{35,42}$. The vdW gaps between the bottom layers still exist. Hence, to improve the carrier injection into the bottom layers, it is preferable to have edge contacts with all layers.

Although a number of selected metals form strong hybridization at interfaces and therefore suppress the tunnel barrier, Fermi level pinning occurs due to the work function of the metal layer at interfaces changing (into the metal- $\mathrm{MoS}_{2}$ alloy's work function) ${ }^{32}$ as well as the creation of gap states from the weakened intralayer S-Mo bonding ${ }^{45}$. Such effects can significantly impact the Schottky barrier height (SBH).

Apart from 3D metals, one must also consider the possibility of contacting 2D SCs using other (or the same) low-dimensional materials. 'Native' chemical bonds are expected at such interfaces. For example, because the carbon family includes both metallic allotropes (such as metallic carbon nanotubes (CNTs), graphene and wide graphene ribbons) and semiconducting allotropes/structures (such as semiconducting CNTs, graphene nanoribbons (GNRs) and vertically biased AB-stacked bilayer graphene), one can first fabricate semiconducting (or metallic) carbon and then tune one side to be metallic (or semiconducting). Relevant theoretical studies include the CNT/graphene interface ${ }^{46}$ (Fig. 3a), the graphene/GNR interface $^{46,47}$ (Fig. 3b) and the monolayer/multilayer graphene interface $^{48}$. The bonds at such interfaces are native $s p^{2}$ carbon-carbon bonds - the same as the bonds inside both the metallic and the semiconducting sides - resulting in a 'seamless' contact between the two.

In a recent study ${ }^{47}$, an all-graphene circuit scheme based on seamless contacts was proposed and evaluated by numerical simulation. The reported seamless contacts, in which both contacts/interconnects (wide graphene) and transistors (GNRs) are envisioned to start from a single sheet of graphene, greatly reduced the contact resistance (down to $0.1 \mathrm{k} \Omega \mu \mathrm{m}$ ) and improved circuit performance in terms of noise margin, speed and power consumption. a

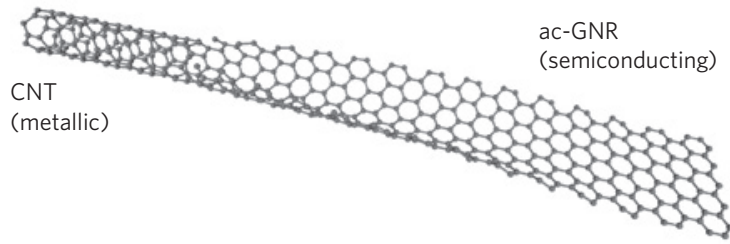

b

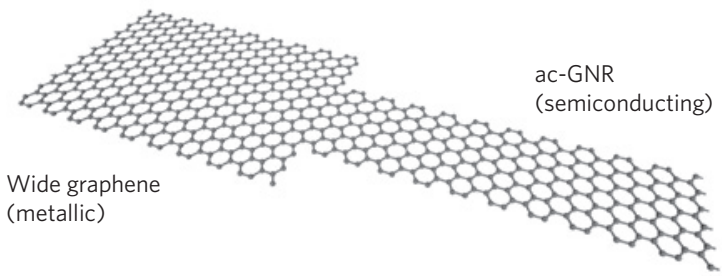

C

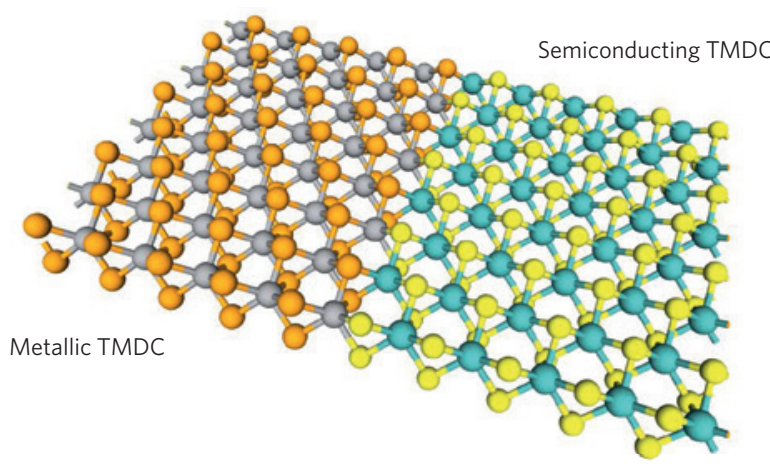

Figure 3 | Various 'seamless' contact schemes. a, CNT-GNR contact. b, Graphene-GNR contact. c, Metallic TMDC-semiconducting TMDC contact. ac, armchair.

The concept of seamless contacts from such all-graphene circuits can also be adapted to other 2D SCs (Fig. 3c). In recent studies ${ }^{49,50}$, contacts between metallic $1 \mathrm{~T}-\mathrm{MoS}_{2}$ and semiconducting $2 \mathrm{H}-\mathrm{MoS}_{2}$ have been fabricated using phase engineering ${ }^{49,51}$ (changing $2 \mathrm{H}$ phase into $1 \mathrm{~T}$ phase). The resulting contact resistance of $0.2 \mathrm{k} \Omega \mu \mathrm{m}$ is the lowest ever reported for this material. Another option would be to grow metallic and semiconducting TMDCs in sequence by chemical vapour deposition. Further theoretical studies are needed to explore this seamless contact scheme on $2 \mathrm{D}$ materials other than graphene.

\section{Charge-injection mechanisms}

There are two mechanisms by which charge can be injected into a SC: thermionic emission over the SB, and field emission (tunnelling) across the SB (Fig. 4a). The thermionic emission-diffusion theory ${ }^{52}$ describes the current-voltage characteristics of a metal-SC junction as a function of SBH. Carrier recombination can also be a current-limiting process if an inversion layer is present near the contact. This is mostly the case in low-bandgap SCs (easy to form an inversion layer) such as Ge nanowires ${ }^{53}$, and could be significant in black phosphorus (which has a small bandgap of $0.3 \mathrm{eV}$ ).

In TMDCs we deal mostly with thermionic emission at low doping, with thermionic field emission starting to contribute as doping increases (Fig. 4a). This is similar to the case of small geometry silicide contacts in advanced complementary metal-oxide-semiconductor (CMOS) technologies ${ }^{54}$.

Contrary to the bulk case (Fig. 2a,b), where the diffusion region B extends both laterally and vertically into the SC, in a metal-2D SC junction with no hybridization (Fig. 2c,d and Fig. 4a), the position of the bands vary only laterally, so that charge carriers injected far 
a

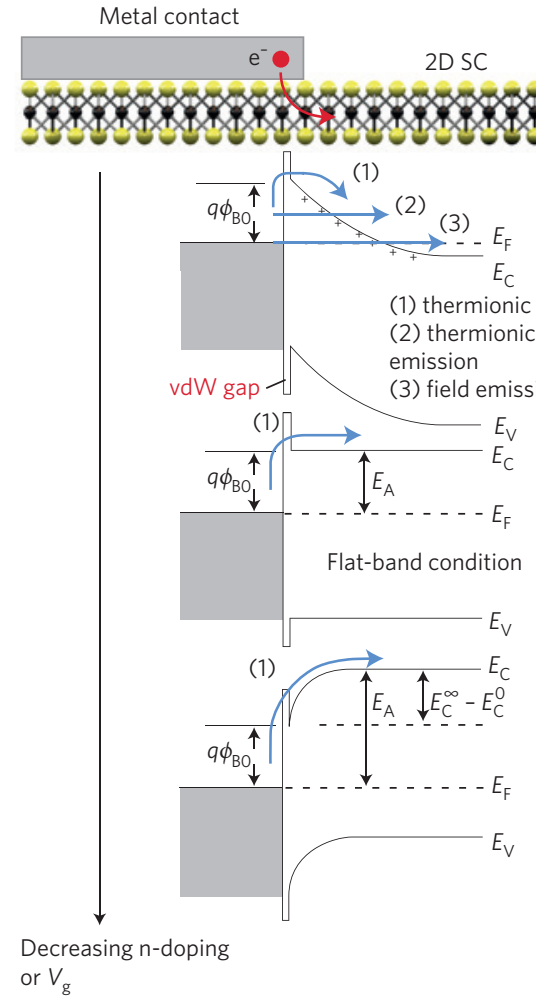

b
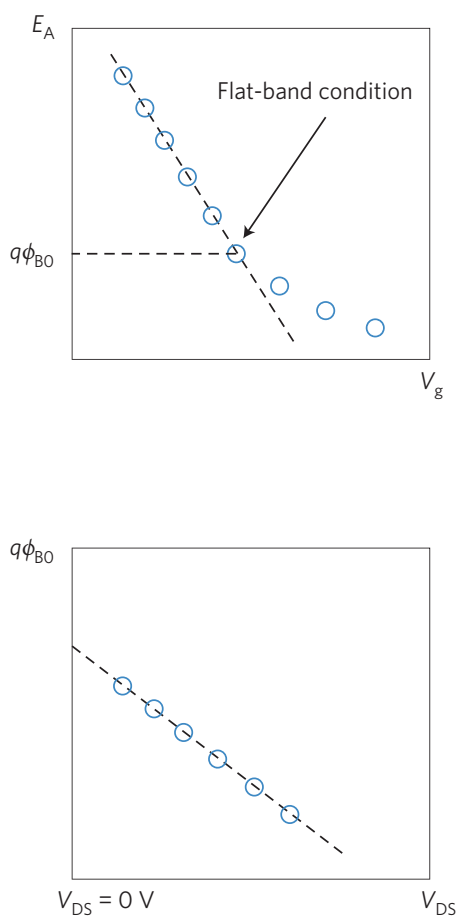

Figure 4 | Charge-injection mechanisms and extraction of the $\mathbf{S B H}$. a, Here we consider the case of a vdW gap (that is, without strong hybridization). Shown are energy diagrams at the end of the charge-injecting source contact (without the B region shown in Fig. $2 \mathrm{~d}$ ), going from high to low channel doping (decreasing gate voltage), as indicated by the vertical black arrow. In the 'off' state, the energy barrier to overcome ( $E_{\mathrm{A}}$ ) is larger than the $\mathrm{SBH}$ $\left(q \phi_{\mathrm{BO}}\right)$. By increasing the doping level, a flat-band condition is met when $E_{\mathrm{A}}=q \phi_{\mathrm{BO}}$. When the channel is doped beyond the flat-band condition, charge injection is mediated by a combination of thermionic emission and tunnelling. $\mathbf{b}$, Method of extracting the SBH. Using transport measurements, $E_{\mathrm{A}}$ is determined for each value of the gate voltage $V_{\mathrm{g}}$ (blue circles). The flat-band condition is realized at the point where $E_{\mathrm{A}}$ stops depending linearly on $V_{\mathrm{g}}$ (dashed line). $E_{\mathrm{A}}$ is then equal to the $\mathrm{SBH} q \phi_{\mathrm{BO}}$. $\mathbf{c}, \mathrm{SBH}$ lowering at finite source-drain bias $V_{\mathrm{DS}}$ due to image forces leads to an underestimation of the $\mathrm{SBH}$. The error can be decreased by extracting the $\mathrm{SBH}$ at finite bias (blue circles) and then extrapolating it to zero bias (dashed line).

from the contact edge first encounter the flat-band region B before the diffusion region $\mathrm{B}^{\prime 32}$ (Fig. $2 \mathrm{c}$, d). In this case, the relative contributions from thermionic emission and tunnelling become difficult to predict.

Because charge injection in 2D SCs strongly depends on the SBH, knowledge of its value and how to alter it would allow the process to be optimized. In the ideal case, the $\mathrm{SBH} q \phi_{\mathrm{B} 0}$ between a metal and a SC is determined by the difference $q \phi_{\mathrm{B} 0}=q\left(\phi_{\mathrm{m}}-\chi\right)$ between the metal's work function $q \phi_{\mathrm{m}}$ and the SC's electron affinity $q \chi$, which is also referred to as the Schottky-Mott $\operatorname{rule}^{52}$ ( $q$ is the elementary electric charge). In reality, however, the Fermi level at the metal/SC interface is often pinned. We can quantify this by inspecting the $\mathrm{SBH}$ dependence on $\phi_{\mathrm{m}}$, given by $S=\mathrm{d} \phi_{\mathrm{B} 0} / \mathrm{d} \phi_{\mathrm{m}}$, with $S=1$ corresponding to the ideal case or Schottky limit, and $S \approx 0$ corresponding to that of a pinned Fermi level. The origin of this pinning in metal/bulk SC interfaces is the presence of metal-induced gap states ${ }^{55}$. For metal contacts to 2D SCs, as discussed in previous sections, the presence of a metal- $\mathrm{MoS}_{2}$ alloy with a different work function ${ }^{32}$ and the creation of gap states from the weakened intralayer S-Mo bonding ${ }^{45}$ contribute to the pinning. The resulting reduced tunability of the $\mathrm{SBH}$ reduces the efficacy of engineering ohmic contacts by choice of the contact metal (or work function) alone.

\section{Extraction of the Schottky barrier height}

The most common way of extracting the $\mathrm{SBH}$ is to measure the activation energy in the thermionic emission regime. In a SB FET geometry, two Schottky diodes connected back-to-back are situated at the source and drain. The reverse-biased contact (the source side of an n-type SB FET; Fig. 4a) consumes most of the voltage drop and dominates the transistor behaviour. The current density injected through a reverse-biased SB is:

$$
J=A^{*} T^{\alpha} \exp \left[-\frac{q \phi_{\mathrm{B} 0}}{k_{\mathrm{B}} T}\right]\left[1-\exp \left(-\frac{q V}{k_{\mathrm{B}} T}\right)\right]
$$

where $A^{*}$ is the Richardson constant and can be derived for 3D and 2D SCs, $\phi_{\mathrm{B} 0}$ is the SBH, $\alpha$ is an exponent equal to 2 for bulk SCs and $3 / 2$ for $2 \mathrm{D}$ SCs, $V$ is the applied bias ${ }^{52,56}, T$ is temperature and $k_{\mathrm{B}}$ is the Boltzmann constant. For $q V \gg k_{\mathrm{B}} T$, equation (1) simplifies to:

$$
J=A^{*} T^{\alpha} \exp \left[-\frac{q \phi_{\mathrm{B} 0}}{k_{\mathrm{B}} T}\right]
$$

The transistor behaviour in the sub-threshold regime (small gate voltage, $V_{\mathrm{g}}$ ) (Fig. 4a, bottom) therefore follows ${ }^{57}$ :

$$
J=A^{\star} T^{\alpha} \exp \left[-\frac{E_{\mathrm{A}}}{k_{\mathrm{B}} T}\right]
$$

where $E_{\mathrm{A}}=q \phi_{\mathrm{B} 0}+E_{\mathrm{C}}^{\infty}-E_{\mathrm{C}}^{0}$ is the total activation energy that charge carriers must overcome to access the channel, and $E_{\mathrm{C}}^{\infty}-E_{\mathrm{C}}^{0}$ is the difference between the conduction band minimum in the bulk and at the interface (Fig. 4a), due to upward band bending. The so-called 


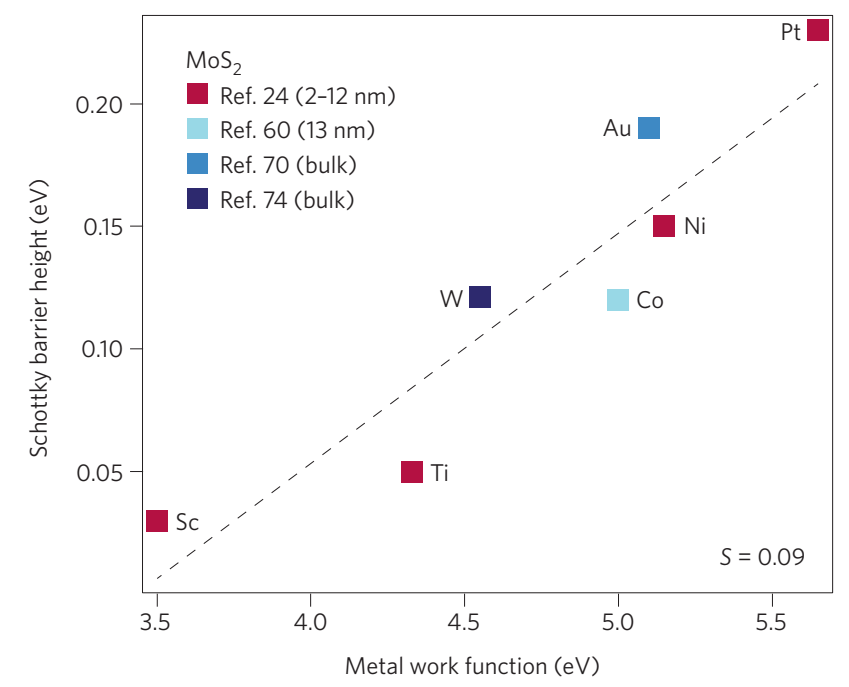

Figure 5 | SBHs for different multilayer $\mathrm{MoS}_{2}$ devices reported in the literature.

flat-band condition $E_{\mathrm{C}}^{\infty}-E_{\mathrm{C}}^{0}=0$ is realized when $V_{\mathrm{g}}=V_{\mathrm{FB}}$ (Fig. 4a, centre band diagram), where $V_{\mathrm{FB}}$ is the flat-band voltage. As long as $V_{\mathrm{g}}<V_{\mathrm{FB}}$, the activation energy $E_{\mathrm{A}}$ depends linearly on $V_{\mathrm{g}}$ :

$$
E_{\mathrm{A}}=q \phi_{\mathrm{B} 0}-\left(1+C_{\mathrm{it}} / C_{\mathrm{ox}}\right)^{-1}\left(V_{\mathrm{g}}-V_{\mathrm{FB}}\right)
$$

where $C_{\text {ox }}$ is the gate oxide capacitance and $C_{\text {it }}$ is the interface trap capacitance ${ }^{24}$.

Increased doping pushes the system from the flat-band condition into a regime where tunnelling contributes (Fig. $4 \mathrm{a}$, top). In this regime, the temperature dependence should deviate from the above equations and $E_{\mathrm{A}}$ will no longer depend linearly on $V_{\mathrm{g}}$.

To extract the $\mathrm{SBH}$, we identify the voltage at which $E_{\mathrm{A}}$ stops depending linearly on $V_{\mathrm{g}}$ (Fig. $4 \mathrm{~b}$ ), given by $E_{\mathrm{A}}=q \phi_{\mathrm{B} 0}$. This method was used in ref. 24 to extract the SBH between $\mathrm{MoS}_{2}$ and different metals. A variant of this method takes care (to first order) of SB lowering by using ${ }^{58}$ :

$$
J=A^{*} T^{\alpha} \exp \left[-\frac{q}{k_{\mathrm{B}} T}\left(\phi_{\mathrm{B} 0}-V / n\right)\right]
$$

where the ideality factor $n$ accounts for barrier lowering due to image charges. The activation barrier at zero bias is then linearly extrapolated from the activation energies obtained at finite biases (Fig. 4c). Several studies have used this second method for $\mathrm{MoS}_{2}$ (refs 58-61).

When using this method, it is important to remember that the activation energy and the SB are not the same thing. They can be identified only when the flat-band condition is met, and not for every value of $V_{\mathrm{g}}$. The channel resistance ${ }^{62}$ contributes significantly to the observed temperature dependence. When the $\mathrm{MoS}_{2}$ channel is gated beyond the metal-insulator transition, its resistance lowers with decreasing temperature ${ }^{63}$. The total (channel plus contact) resistance can then also decrease - this should not be misinterpreted as a negative SB.

The SBH can also be extracted in the thermionic field emission regime. This method was used in refs 64,65 and seems to describe quite well the injection of electrons into $\mathrm{WSe}_{2}$ from ionic-liquidgated contacts ${ }^{64}$. Other methods that have been used to extract the $\mathrm{SBH}$ in 2D SCs include ultraviolet and X-ray photoelectron spectroscopy ${ }^{66-69}$, which measures the band bending, and scanning tunnelling spectroscopy of Au nanoparticles deposited on top of $\mathrm{MoS}_{2}$ (refs 70,71). Finally, some techniques that could in future

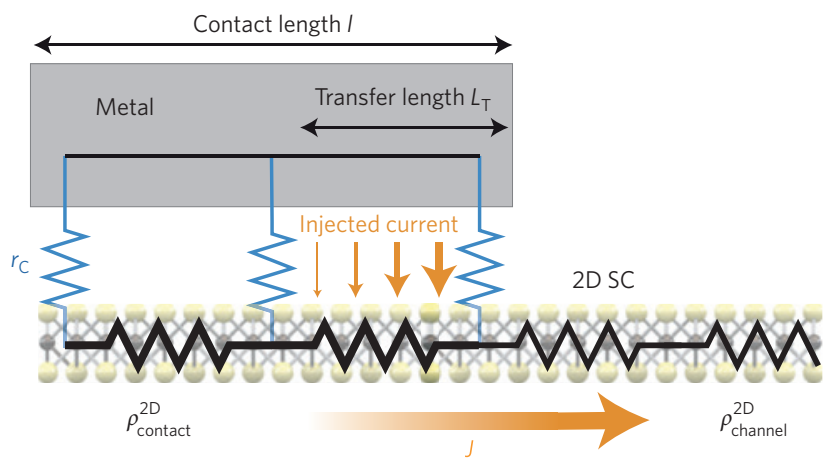

Figure 6 | Current crowding at the contact edge region. The resistor network illustrates the transmission line model. The orange arrows depict the current density, J. $r_{\mathrm{C}}$ represents the resistivity of the metal/SC interface. $\rho_{\text {contact }}^{2 D}$ and $\rho_{\text {channel }}^{2 D}$ represent the SC's sheet resistivity under the contact and in the channel, respectively.

be employed for TMDCs include internal photoemission ${ }^{72,73}$ and $C-V$ measurements ${ }^{30}$.

Figure 5 plots $\mathrm{SBH}$ values between multilayer $\mathrm{MoS}_{2}$ and different metals ${ }^{24,60,70,74}$ against the corresponding metal work functions. In the limit of 1-3 layers, the SBH is expected to depend strongly on the number of layers because the bandgap increases as the thickness is decreased. Figure 5 therefore only compares values of $\mathrm{SBH}$ that were obtained on thicker (from $2 \mathrm{~nm}$ to bulk) layers. As was shown in ref. 24, in this range there is no significant dependence of the $\mathrm{SBH}$ on $\mathrm{MoS}_{2}$ thickness. It can be seen from Fig. 5 that the Fermi level is pinned to around $100 \mathrm{meV}$ below the conduction band, and that the SBH depends weakly on the metal's work function $(S=0.09)$. This is in contradiction with early results from $\mathrm{X}$-ray photoelectron spectroscopy measurements, which indicated that the SBH for bulk $\mathrm{MoS}_{2}$ is in the Schottky limit $(S=1)^{66}$.

A recent scanning tunnelling microscopy investigation offers one possible explanation for this discrepancy ${ }^{75}$. The $\mathrm{MoS}_{2}$ surface hosts a high density of metallic defects that are likely to be clusters of sulphur vacancies. These defects are associated with variations in the Fermi level as large as $1 \mathrm{eV}$ and are believed to shunt the pristine regions and thus strongly influence electrical properties. The SBH values derived from electrical measurements can therefore be significantly lower than those measured with ultraviolet and X-ray photoelectron spectroscopy. These observations are also in line with previous observations of widely varying $\mathrm{SBH}$ in $\mathrm{Au}$ nanoclusters evaporated onto $\mathrm{MoS}_{2}$ (ref. 71). SBH inhomogeneities can also explain ${ }^{76}$ the apparent temperature dependence of $\mathrm{SBH}$ and ideality factor in graphene- $\mathrm{MoS}_{2}$ (ref. 77) and In-WSe (ref. 78) contacts.

$\mathrm{WSe}_{2}$ was also investigated by scanning tunnelling microscopy $^{79}$ and, although similar structural defects were present, no metallic defects were found. This could explain why contacts to $\mathrm{WSe}_{2}$ are often much more resistive. Ultraviolet and X-ray photoelectron spectroscopy analysis has revealed that, like $\mathrm{MoS}_{2}$, the $\mathrm{SBH}$ for $\mathrm{WSe}_{2}$ approaches the Schottky limit ${ }^{67-69}$ - a fact supported by the high sensitivity of the SBH to graphene's work function in graphene-WSe contacts $^{64}$.

Decoupling the metal from the SC surface decreases the amount of Fermi level pinning and has a number of positive effects. It was recently reported that an intervening oxide ${ }^{58,60,61,80}$ or graphene $e^{81}$ layer between the metal and $\mathrm{MoS}_{2}$ significantly reduces the contact resistance and $\mathrm{SBH}$.

Fermi level pinning is also critical because it impacts the possibility of realizing FETs with both polarities. FETs made of (naturally n-doped) mono- and multilayer $\mathrm{MoS}_{2}$ exhibit n-type behaviour with all metals, with the notable exception of $\mathrm{MoO}_{x}$ contacts to 
a

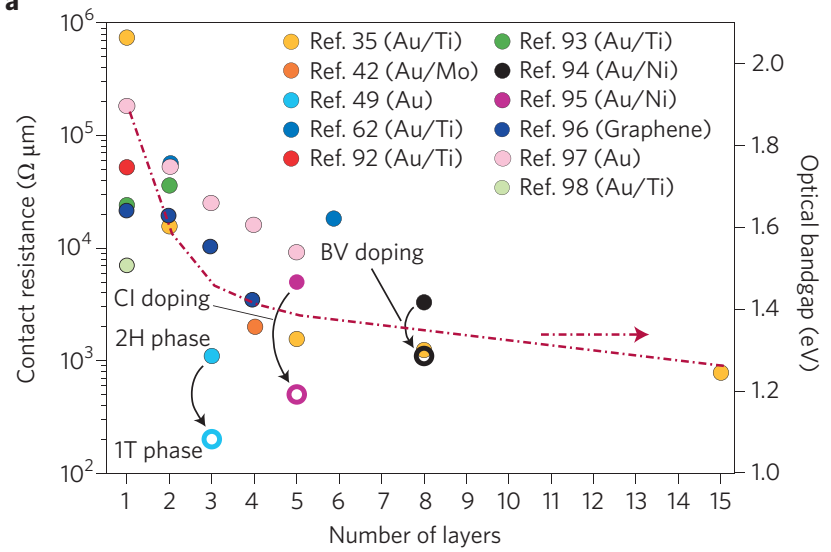

b

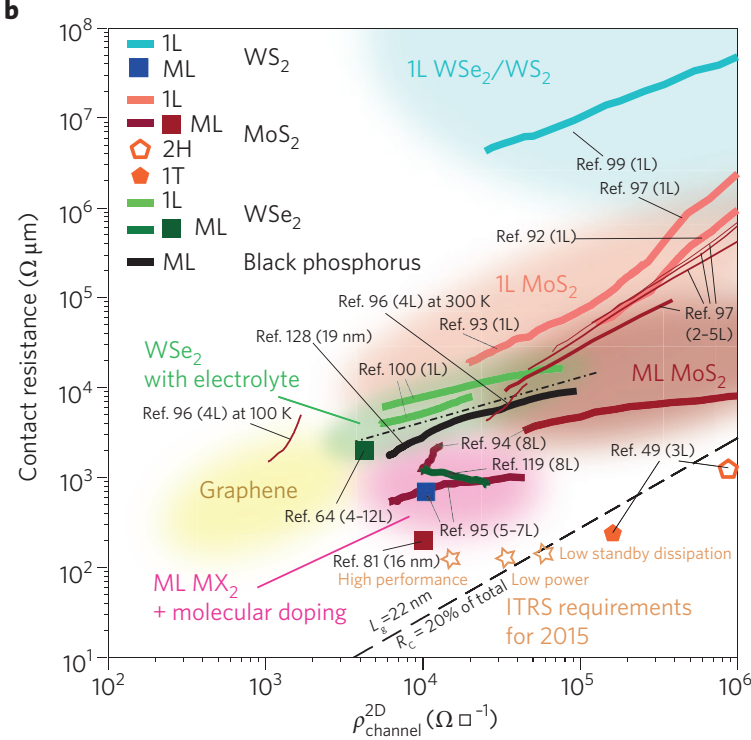

Figure 7 | Contact resistance for top-contacted 2D SCs. a, Minimum contact resistance as a function of the number of atomic layers in several studies on $\mathrm{MoS}_{2}$ (refs 35,42,49,62,92-98). The red dashed line depicts the optical bandgap measured in ref. 9. b. Contact resistance for different values of channel sheet resistivity in several different studies on TMDCs, black phosphorus and graphene ${ }^{49,64,81,92-96,99,100,119,97,128}$. The dashed line depicts the criterion $R_{\mathrm{C}}=20 \%$ of total resistance in a transistor with a 22 -nm-long gate, $L_{g}$. The dash-dot line is a guide to the eye: if a line runs parallel to it, its $R_{\mathrm{C}}$ scales as $\sqrt{ } \rho_{\text {channel. }}^{2 D}$. The different star symbols represent the target values for 2015 as indicated by ITRS 2012 for different types of transistor, namely high performance, low power and low standby dissipation.

multilayer $\mathrm{MoS}_{2}$ (ref. 82). In WSe $\mathrm{WETs}_{2}$ the situation is more contrasted, with reports of clear n-type transport in monolayers with $\mathrm{Al}, \mathrm{Ti}, \mathrm{Ag}$, In (ref. 6) and $\mathrm{Au}$ (ref. 83), and clear p-type transport with $\mathrm{Au}$ (ref. 84), Pd (ref. 85) and $\mathrm{Ag}$ (ref. 86). Finally, owing to its very high work function, $\mathrm{MoO}_{x}$ seems to be the best hole injector for both multilayer $\mathrm{MoS}_{2}$ and WSe 2 (refs 79,82).

\section{Contact resistance scaling}

The top contact is the most common contact geometry in use today. In the weak-coupling limit, the contact resistance is a combination of the metal/SC interface resistivity $r_{\mathrm{C}}$ (expressed in ohms square metre; $\Omega \mathrm{m}^{2}$ ) and the SC sheet resistivity $\rho^{2 \mathrm{D}}$ (expressed in ohms per square or $\left.\Omega \square^{-1}\right)^{87}$. If the contact is diffusive - that is, if the charge carriers are scattered many times within the SC before being kicked out of the SC and into the metal - then the interface can be modelled as a resistor network (Fig. 6). This is the so-called transmission line model ${ }^{88,89}$, which gives the following expression for the contact resistance $R_{\mathrm{C}}$ (in $\Omega \mathrm{m}$ ):

$$
R_{\mathrm{C}}=\sqrt{\rho^{2 \mathrm{D}} r_{\mathrm{C}}} \operatorname{coth}\left(l \sqrt{\frac{\rho^{2 \mathrm{D}}}{r_{\mathrm{C}}}}\right)
$$

where $l$ is the contact length. It can be seen that the dependence of $R_{\mathrm{C}}$ on $l$ is nonlinear because of current crowding. For contact lengths much larger than the transfer length $L_{\mathrm{T}}=\sqrt{ }\left(r_{\mathrm{C}} / \rho^{2 \mathrm{D}}\right)$, where $L_{\mathrm{T}}$ is the average distance that an electron (or hole) travels in the SC beneath the contact before it enters the contact, the expression for the contact resistance becomes $R_{\mathrm{C}}=\sqrt{ }\left(\rho^{2 \mathrm{D}} r_{\mathrm{C}}\right)$ (in units of $\Omega \mathrm{m}$ ) and is no longer dependent on the contact length. In some reports the 'as-measured' resistance is referred to as the 'contact resistance', in which case the so-called contact resistivity is expressed in units of $\Omega \mathrm{m}$. The quantity 'resistance $\times$ contact area' (in units of $\Omega \mathrm{m}^{2}$ ) is sometimes also used to characterize contacts, especially when contacts exhibit a significant dependence on contact length $l$ (when $l$ and $L_{\mathrm{T}}$ are of the same order of magnitude) ${ }^{90}$.

In graphene, the diffusive approximation breaks down due to the longer electron mean free path, which calls for a ballistic treatment of the contact resistance ${ }^{91}$. In TMDCs, the much lower mean free path implies that the transmission line model can be applied, provided that the resistivity of the portion of semiconducting material under the contact $\left(\rho_{\text {contact }}^{2 \mathrm{D}}\right)$, and not the resistivity of the semiconducting channel $\left(\rho_{\text {channel }}^{2 \mathrm{D}}\right)$, is used in place of $\rho^{2 \mathrm{D}}$ in equation (6). Note, however, that this approach cannot accurately model metal contacts to multilayers owing to the greater impact of edge contacts ${ }^{36,37}$.

The transfer length has been studied in monolayer ${ }^{92}$ and $2 \mathrm{~L}-6 \mathrm{~L}$ (ref. 62) $\mathrm{MoS}_{2}$. A value of $L_{\mathrm{T}}=600 \mathrm{~nm}$ was found ${ }^{92}$ in the monolayer case, assuming $\rho_{\text {contact }}^{2 \mathrm{D}}=\rho_{\text {channel. }}^{2 \mathrm{D}}$. Using a more elaborate measurement ${ }^{62}$ similar to a four-terminal Kelvin resistor scheme described in ref. 87, one can accurately determine both $\rho_{\text {contact }}^{2 \mathrm{D}}$ and $r_{\mathrm{C}}$. A value of $L_{\mathrm{T}}=20-70 \mathrm{~nm}$ was found for bilayer $\mathrm{MoS}_{2}$ with Ti contacts and up to $200 \mathrm{~nm}$ in $6 \mathrm{~L} \mathrm{MoS}_{2}$ (ref. 62). This discrepancy indicates that the assumption $\rho_{\text {contact }}^{2 \mathrm{D}}=\rho_{\text {channel }}^{2 \mathrm{D}}$ fails in the case of atomically thin channels owing to the strong influence of the metallic electrode on the channel underneath.

\section{Experimental review of contact resistances}

Figure 7 shows the contact resistances of 2D SCs found in the literature. From an experimental point of view, the contact resistance depends mainly on three parameters: contact metal, $\rho_{\text {contact }}^{2 \mathrm{D}}$ and number of layers. This makes it difficult to compare contact resistance values found in the literature because available data sets often differ by more than one parameter. Although results obtained using different metals are available, from this meta-analysis it is difficult to draw clear conclusions as to which metal yields the best contact to any given material. We nonetheless indicate the contact metal used in each study. Figure $7 \mathrm{a}$ shows the minimal $R_{\mathrm{C}}$ values from several studies on $\mathrm{MoS}_{2}$ as a function of number of layers ${ }^{35,42,49,62,92-98}$. Despite the scatter in the data, there is a clear trend of decreasing $R_{\mathrm{C}}$ with increasing thickness. This comes as no surprise because the larger bandgap in thinner flakes (red dashed line in Fig. 7a) is expected to give rise to larger SBs.

A better comparison involves contact resistances measured at similar sheet resistances. We do not have access to the values of $\rho_{\text {contact }}^{2 \mathrm{D}}$, but using the channel resistivity $\rho_{\text {channel }}^{2 \mathrm{D}}($ Fig. $7 \mathrm{~b})$ instead should provide a more reliable comparison between contacts. Each curve in Fig. 7b corresponds to a back-gate voltage sweep, wherein the back gate dopes both the channel and the contact areas. We assembled data points from a set of studies reporting both contact resistance and total transistor resistance (in some cases four-probe resistance), and in which $L \gg L_{\mathrm{T}}$, so that contact length is irrelevant. The channel resistivity was extracted by subtracting the contact 
a

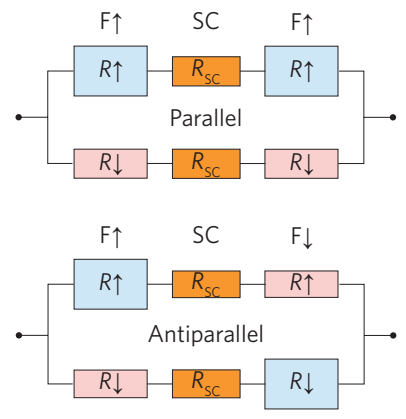

c

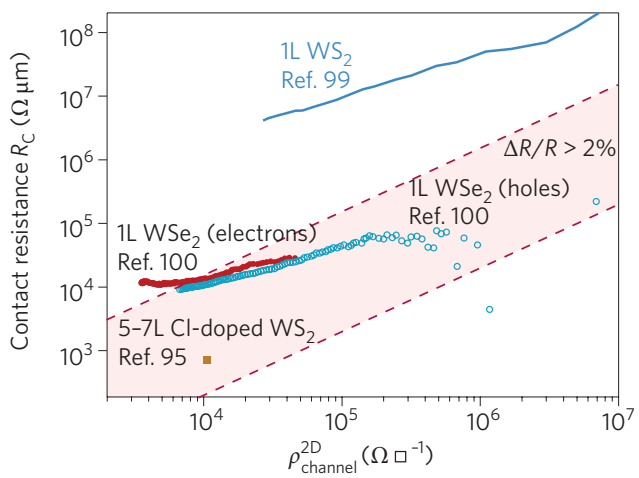

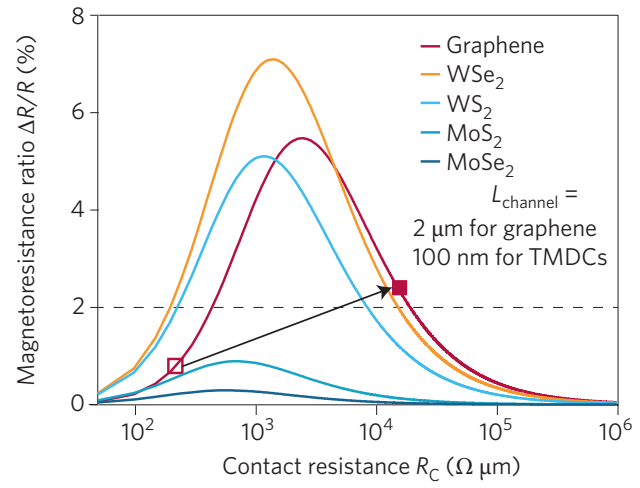

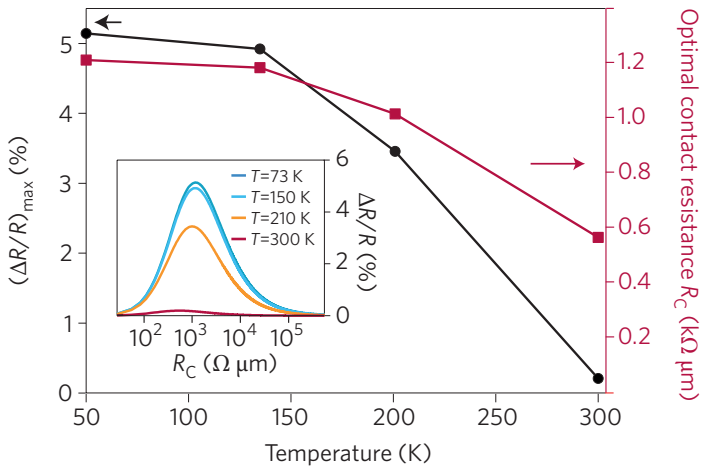

Figure $\mathbf{8}$ | Spin injection and contact resistance. a, In devices composed of SCs contacted with ferromagnetic (F) contacts, the magnetoresistance signal vanishes when the resistance of the nonmagnetic material is much higher than that of the contacts. $\mathbf{b}$, Magnetoresistance as a function of contact resistance calculated from equation (9) (Box 1) for parameters listed in Table 1. The filled and empty squares indicate where the contact resistance of the ferromagnet-graphene contact lies with and without a tunnel barrier, respectively ${ }^{116}$. c. Contact resistance as a function of channel sheet resistivity in WSe ${ }_{2}$

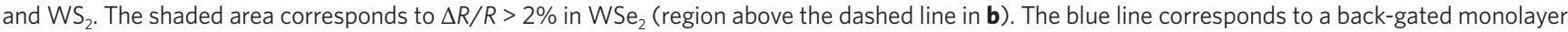
$\mathrm{WS}_{2}$ device ${ }^{99}$. The red and blue dots were obtained in electrolyte-gated $1 \mathrm{~L}$ (monolayer) $\mathrm{WSe}_{2}$. The square is a multilayer $\mathrm{WS}_{2}$ with molecular doping ${ }^{95}$. d. Calculated maximum magnetoresistance as a function of temperature in monolayer $\mathrm{WS}_{2}$ and corresponding optimal contact resistance calculated using experimentally determined temperature-dependent mobilities ${ }^{99}$ and spin lifetimes ${ }^{124}$. Inset: Magnetoresistance as a function of contact resistance.

resistance from the total resistance and renormalizing with the channel's aspect ratio: $\rho_{\text {channel }}^{2 \mathrm{D}}=\left(R_{\text {total }}-R_{\text {contacts }}\right) W / L$, where $L$ and $W$ are the length and width of the channel, respectively

We plot the results obtained on different TMDCs and black phosphorus, mono- and multilayer, before and after molecular or electrolyte gating of the channel, as well as the 2015 silicon-on-insulator target values according to the 2012 International Technology Roadmap for Semiconductors (ITRS). The significance of this representation is evident if one compares the data from ref. 92 and ref. 93: in Fig. 7a, the minimum $R_{\mathrm{C}}$ (monolayer case) seems to be lower for ref. 93, but Fig. $7 \mathrm{~b}$ reveals that this is only due to higher doping. Actually, for a given value of sheet resistivity, $R_{\mathrm{C}}$ is lower in ref. 92 .

Figure $7 \mathrm{~b}$ reveals several interesting things. First, it confirms that, at constant sheet resistivity, the contact resistance decreases with increasing $\mathrm{MoS}_{2}$ thickness ${ }^{97}$. We can also see that in many cases (thick $\mathrm{MoS}_{2}, \mathrm{WS}_{2}, \mathrm{WSe}_{2}$ and black phosphorus), $R_{\mathrm{C}}$ scales as $\sqrt{ } \rho_{\text {channel }}^{2 \mathrm{D}}$ (dash-dot line), which indicates that the transmission line model (equation (6)) applies reasonably well even if we use $\rho_{\text {channel }}^{2 \mathrm{D}}$ instead of

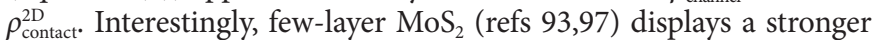
dependence on $\rho_{\text {channel }}^{2 \mathrm{D}}$, compared with multilayers ${ }^{94,97}$. This could indicate that, in this particular case, the assumption $\rho_{\text {contact }}^{2 \mathrm{D}} \approx \rho_{\text {channel }}^{2 \mathrm{D}}$ is not valid because of possible $\mathrm{MoS}_{2}$ band structure distortion by metal orbitals and Fermi level pinning under the contacts. In comparison, monolayer $\mathrm{WS}_{2}$ with Au contacts ${ }^{99}$ displays a much higher $R_{\mathrm{C}}$ but it follows roughly $\sqrt{ } \rho_{\text {channel }}^{2 \mathrm{D}}$, which may indicate a weaker coupling to the metal compared with $\mathrm{MoS}_{2}$.

Molecular and electrolyte doping of the channel have often been used to decrease the contact resistance ${ }^{94,95}$ (Fig. 7a). Figure $7 \mathrm{~b}$ shows that this effect is partially due to decreased channel resistivity. Yet this cannot be the only effect, otherwise data before and after doping would fall on a straight line. This reflects the enhanced contribution from tunnelling at the contact edges as the SB is thinned down, which seems particularly strong in $\mathrm{WSe}_{2}$ (refs 64,100).

By far, the lowest contact resistances have been obtained using phase engineering on few- ${ }^{49}$ and single-layer ${ }^{50} \mathrm{MoS}_{2}$. The local conversion of $\mathrm{MoS}_{2}$ from the semiconducting $2 \mathrm{H}$ phase to the metallic $1 \mathrm{~T}$ phase under the metal contacts results in seamless contacts with resistances as low as $200 \Omega \mu \mathrm{m}$ in very resistive devices, thus pushing the performance of TMDC-based transistors closer to the quantum limit ${ }^{27-29}$.

Graphene is a promising candidate to contact 2D SCs ${ }^{64,101-103}$, and it can be used to realize purely $2 \mathrm{D}$ circuits ${ }^{103}$. Another advantage of graphene is the tunability of its work function by electrostatic doping, which makes it a versatile contact metal ${ }^{64}$. The interface between $\mathrm{MoS}_{2}$ and graphene was recently found ${ }^{96}$ to yield very low $R_{\mathrm{C}}$, as well as reducing $R_{\mathrm{C}}$ with decreasing temperature in ultraclean $\mathrm{MoS}_{2}$ samples. Once again, this result can be understood in the framework of the transmission line model: in these high-mobility devices, the sheet resistivity drops by several orders of magnitude as the temperature is lowered, which in turn affects the contact resistance. Figure $7 \mathrm{~b}$ shows the data from ref. 96 at $T=300 \mathrm{~K}$ and $T=100 \mathrm{~K}$. The two data sets can be roughly linked by the dash-dot line, which indicates that equation (6) describes the decreased contact resistance. When compared with other metals at comparable sheet resistivity ${ }^{97}$, however, graphene performs well but is not significantly better. One intrinsic limitation of graphene-TMDC contacts 


\section{Box 1 | Magnetoresistance of a 2D SC-based lateral spin valve.}

Introducing the resistivity of the ferromagnetic electrodes $\rho_{\mathrm{F}}$, contact resistance $r_{\mathrm{C}}$ and the spin asymmetry coefficients $\beta$ and $\gamma$ of the electrodes and tunnel barriers, respectively, defined as $\rho_{\mathrm{F}}^{\uparrow(\downarrow)}=2 \rho_{\mathrm{F}}^{\star}(1-(+) \beta)$ and $r_{\mathrm{C}}^{\uparrow(\downarrow)}=2 r_{\mathrm{C}}^{\star}(1-(+) \gamma)$, where $\rho_{\mathrm{F}}=\rho_{\mathrm{F}}^{\star}\left(1-\beta^{2}\right)$ and $r_{\mathrm{C}}=r_{\mathrm{C}}^{\star}\left(1-\gamma^{2}\right)$, the relative magnetoresistance $\Delta R / R$ is given by ${ }^{113}$ :

$$
\Delta R=\frac{2\left(\beta r_{\mathrm{F}}+\gamma r_{\mathrm{C}}^{*}\right)^{2}}{\left(r_{\mathrm{C}}^{*}+r_{\mathrm{F}}\right) \cosh \left(\frac{L}{l_{\mathrm{sf}}^{\mathrm{SC}}}\right)+\frac{r_{\mathrm{SC}}}{2}\left[1+\left(\frac{r_{\mathrm{C}}^{*}}{r_{\mathrm{SC}}}\right)^{2}\right] \sinh \left(\frac{L}{l_{\mathrm{sf}}^{\mathrm{SC}}}\right)}
$$

and

$$
\begin{aligned}
R=2\left(1-\beta^{2}\right) r_{\mathrm{F}}+r_{\mathrm{SC}} \frac{L}{l_{\mathrm{sf}}^{\mathrm{SC}}}+2\left(1-\gamma^{2}\right) r_{\mathrm{C}}^{*}+ \\
2 \frac{(\beta-\gamma)^{2} r_{\mathrm{F}} r_{\mathrm{C}}^{*}+r_{\mathrm{SC}}\left(\beta^{2} r_{\mathrm{F}}+\gamma^{2} r_{\mathrm{C}}^{*}\right) \tanh \left(\frac{L}{2 l_{\mathrm{sf}}^{\mathrm{SC}}}\right)}{\left(r_{\mathrm{F}}+r_{\mathrm{C}}^{*}\right)+r_{\mathrm{SC}} \tanh \left(\frac{L}{2 l_{\mathrm{sf}}^{\mathrm{SC}}}\right)}
\end{aligned}
$$

where $r_{\mathrm{F}}=\rho_{\mathrm{F}}^{\star} l_{\mathrm{sf}}^{\mathrm{F}}$ and $r_{\mathrm{SC}}=\rho_{\mathrm{SC}} l_{\mathrm{sf}}^{\mathrm{SC}}$ have units of $\Omega \mathrm{m}^{2}, l_{\mathrm{sf}}^{\mathrm{SC}}$ and $l_{\mathrm{sf}}^{\mathrm{F}}$ are the spin diffusion lengths in the $\mathrm{SC}$ and ferromagnet, respectively $\left(l_{\mathrm{sf}}=\sqrt{ } D \tau_{\mathrm{sf}}\right.$, where $D=\mu k_{\mathrm{B}} T / q$ is the spin diffusivity, $\tau_{\mathrm{sf}}$ is the spinflip time and $\mu$ is the electron mobility), and $L$ is the length of the SC channel.

The spin injection efficiency, given by $\gamma=\left(J_{\uparrow}-J_{\downarrow}\right) /\left(J_{\uparrow}+J_{\downarrow}\right)$, depends on both the polarization of the ferromagnet's density of states and the amount of spin-flip during tunnelling. This can be

is that the vdW gap between graphene and $\mathrm{MoS}_{2}$ is about $0.33 \mathrm{~nm}$, as shown by DFT simulations ${ }^{104}$, which is larger than that in typical metal- $\mathrm{MoS}_{2}$ contacts (such as $0.15 \mathrm{~nm}$ in $\mathrm{Ti}-\mathrm{MoS}_{2}$; ref. 32).

Graphene can also be used as a buffer layer between $\mathrm{MoS}_{2}$ and a bulk metal. It has been demonstrated ${ }^{81}$ that using a layer of graphene between multilayer $\mathrm{MoS}_{2}$ and $\mathrm{Ni}$ contacts significantly reduces the $\mathrm{SBH}$, resulting in a 20 -fold improvement of the contact resistance and yielding one of the best-ever reported contact resistances with TMDCs. This promising result calls for more experiments, in particular with single- and few-layer TMDCs. Finally, note that although excellent contact resistances were recently achieved by edge-contacting graphene ${ }^{31}$, so far the only reported attempts of edge-contacting TMDCs have produced very resistive contacts ${ }^{105}$.

As we have seen, when scaling devices we must consider the existence of a finite 'transfer length' within which charges are injected. Another limitation comes from the requirement of 'ohmic' behaviour. For a FET to operate properly and without nonlinearities in the transistor output, the contact resistance $R_{\mathrm{C}}$ should be a small fraction of the total resistance. For example, the ITRS 2012 requirement for low-standby-power silicon-on-insulator FETs, a geometry close to FETs based on 2D SCs, is that the total contact resistance should be down to $20 \%$ of the total resistance by 2015 . As a consequence, $R_{\mathrm{C}}$ should scale with channel length. The dashed black line in Fig. $7 \mathrm{~b}$ depicts this criterion for a channel length of $22 \mathrm{~nm}$. It is noteworthy that the performance of $1 \mathrm{~T}$ contacts ${ }^{49}$ are compliant with the ITRS 22-nm-node requirement.

\section{Spin injection}

Not all applications require minimal contact resistance; a prime example of this is the field of spintronics, in which the criterion for success is impedance matching rather than minimizing contact as low as $1 \%$ in transparent contacts and as high as $35 \%$ in $\mathrm{MgO}$ tunnel barriers on graphene ${ }^{115}$. Note that $\gamma$ might be lower in the case of $\mathrm{SBs}^{118}$.

The expression $\Delta R / R$ can be adapted for the case of a $2 \mathrm{D}$ channel using indications given in ref. 113. When the SC thickness $t$ is no longer comparable to the contact width $w$, relaxation in the SC is divided by a factor $w / t$. We must then modify the expression by using $r_{\mathrm{SC}}=\rho_{\mathrm{SC}} l_{\mathrm{sf}}^{\mathrm{SC}} w / t=\rho_{\text {channel }}^{2 \mathrm{D}} l_{\mathrm{sf}}^{\mathrm{SC}} w$. Knowing that $r_{\mathrm{F}} \ll r_{\mathrm{SC}}, r_{\mathrm{C}}^{*}$ and using $R_{\mathrm{C}}^{*}=r_{\mathrm{C}}^{*} w=R_{\mathrm{C}} /\left(1-\gamma^{2}\right)$, where $R_{\mathrm{C}}$ is the contact resistance in $\Omega \mathrm{m}$, we finally have:

$$
\begin{aligned}
& \frac{\Delta R}{R}=\frac{2\left(\gamma R_{\mathrm{C}}^{*}\right)^{2}}{R_{\mathrm{C}}^{*} \cosh \left(\frac{L}{l_{\mathrm{sf}}^{\mathrm{SC}}}\right)+\frac{\rho_{\text {channel }}^{2 \mathrm{D}} l_{\mathrm{sf}}^{\mathrm{SC}}}{2}\left[1+\left(\frac{R_{\mathrm{C}}^{*}}{\rho_{\text {channel }}^{2 \mathrm{D}} l_{\mathrm{sf}}^{\mathrm{SC}}}\right)^{2}\right] \sinh \left(\frac{L}{l_{\mathrm{sf}}^{\mathrm{SC}}}\right)^{\times}} \\
& {\left[\rho_{\text {channel }}^{2 \mathrm{D}} L+2\left(1-\gamma^{2}\right) R_{\mathrm{C}}^{*}+2 \frac{\rho_{\text {channel }}^{2 \mathrm{D}} l_{\mathrm{sf}}^{\mathrm{SC}} \gamma^{2} R_{\mathrm{C}}^{*} \tanh \left(\frac{L}{2 l_{\mathrm{sf}}^{\mathrm{SC}}}\right)}{R_{\mathrm{C}}^{*} \rho_{\text {channel }}^{2 \mathrm{D}} l_{\mathrm{sf}}^{\mathrm{SC}} \tanh \left(\frac{L}{2 l_{\mathrm{sf}}^{\mathrm{SC}}}\right)}\right]^{-1}}
\end{aligned}
$$

This expression is very similar to that derived in ref. 117 for the case of graphene, except for the contribution from $R_{\text {channel }} \geq R_{\text {contact, }}$, which must be taken into account in our case where $l_{\mathrm{sf}}^{\mathrm{SC}} \leq L$. This expression is valid for a 'confined' geometry, in which the channel does not extend laterally beyond the electrodes. This has been discussed in detail in ref. 117.

resistance. Combining broken inversion symmetry and strong spin-orbit coupling in monolayer TMDCs leads to an interesting spin-split valence band, which enables the electrical manipulation of spins ${ }^{106-108}$ and makes semiconducting TMDCs an interesting material family for spintronics. Calculations have also predicted giant magnetoresistance in $\mathrm{Fe} / \mathrm{MoS}_{2} / \mathrm{Fe}$ sandwich structures ${ }^{109}$. So far, however, the realization of spin injection from ferromagnetic contacts into TMDCs remains elusive. In this section we will briefly discuss the conditions that must be met, with regard to the contacts, to realize spin injection in TMDCs.

The simplest spintronics device is the 'spin valve', in which two ferromagnetic electrodes, bridged by a non-magnetic material, are polarized either parallel or antiparallel to each other. The magnetoresistance ratio $\Delta R / R$ between these two configurations is the figure of merit for this device. The current flow can be represented by a resistor network, with two parallel current paths corresponding to the two spin channels ${ }^{110}$ (Fig. 8a). It can thus easily be seen that the magnetoresistance ratio drops when the resistance of the SC becomes large. The injection of a spin-polarized current into a SC is therefore hampered by the conductivity mismatch between the SC and the (usually metallic) ferromagnetic electrodes ${ }^{111}$.

The most common way around this problem is to modulate the contact resistance by introducing tunnel barriers at the contacts $^{112,113}$. Tunnel barriers also have a spin-dependent resistance and can thus play the role of $R_{\uparrow}$ and $R_{\downarrow}$ in Fig. $8 \mathrm{a}$, with arbitrarily high resistances. However, for very high resistances, the transit time of electrons becomes too long. Because of this, the contact resistance of the barriers must be well adjusted so that a significant magnetoresistance signal can be observed.

The magnitude of the magnetoresistance signal as a function of contact resistance can be calculated using the approach proposed 
Table 1 | Material properties used in calculations of spin injection and contact resistance.

\begin{tabular}{|c|c|c|c|c|c|c|c|c|}
\hline Material & $\rho^{2 \mathrm{D}}\left(\Omega \square^{-1}\right)$ & $T(\mathrm{~K})$ & $\mu\left(\mathrm{cm}^{2} \mathrm{~V}^{-1} \mathrm{~s}^{-1}\right)$ & $D\left(\mathrm{~m}^{2} \mathrm{~s}^{-1}\right)$ & $\tau_{\mathrm{sf}}(\mathrm{ps})$ & $I_{\mathrm{sf}}^{\mathrm{sc}}(\mathrm{nm})$ & $L(\mathrm{~nm})$ & References \\
\hline Graphene & $10^{3}$ & 300 & 7,000 & $1.8 \times 10^{-2}$ & 135 & 1,600 & 2,000 & 116 \\
\hline $\mathrm{MoS}_{2}$ & $10^{4}$ & 74 & 200 & $1.3 \times 10^{-3}$ & 10 & 115 & 100 & 63,125 \\
\hline $\mathrm{MoSe}_{2}$ & $10^{4}$ & 300 & 30 & $7.8 \times 10^{-5}$ & 9 & 26 & 100 & 126 \\
\hline$W_{2}$ & $10^{4}$ & 74 & 100 & $6.4 \times 10^{-4}$ & 88 & 237 & 100 & 99,124 \\
\hline $\mathrm{WSe}_{2}$ & $10^{4}$ & 4 & 250 & $8.6 \times 10^{-6}$ & 1,000 & 92 & 100 & 100,127 \\
\hline
\end{tabular}

in ref. 113 (Box 1). This gives us the magnitude of the 'local' signal. The nonlocal signal can be calculated using the approach described in refs 114,115 . Using experimentally determined values of resistivity and valley lifetimes (since the valley and spin degrees of freedom are locked in monolayer TMDCs ${ }^{106}$ ) (Table 1), with a spin injection efficiency of $\gamma=0.5$, we can estimate the magnetoresistance ratio for different TMDCs using equation (9) in Box 1. Note that these values apply only to out-of-plane spins, which are stabilized by the strong spin-orbit coupling.

The results are shown in Fig. 8b,c. The much larger spin diffusion length in graphene (Table 1) is related to its larger mobility. Nevertheless, Fig. $8 \mathrm{~b}$ shows that a measurable signal (comparable with those in refs 115,116 , in which $L_{\text {channel }}=2 \mu \mathrm{m}$ ) can be expected in reasonably short junctions of $\mathrm{WS}_{2}$ and $\mathrm{WSe}_{2}\left(L_{\text {channel }}=100 \mathrm{~nm}\right)$.

It is noteworthy that graphene and TMDCs lie on two different sides of the conductivity-mismatch problem. In graphene, the contact resistance of direct ferromagnetic contacts is too low, and decoherence occurs mainly through spin escaping into the electrodes. Contacts to TMDCs lie on the right side of the bell, where decoherence occurs inside the channel ${ }^{117}$. In graphene, introducing a tunnel barrier increases the contact resistance and improves the signal $^{115}$. By a fortunate coincidence, the same tunnel barriers tend to decrease the contact resistance to $\mathrm{TMDCs}^{58,60}$.

Figure $8 \mathrm{c}, \mathrm{d}$ focuses on the more promising materials $\mathrm{WSe}_{2}$ and $\mathrm{WS}_{2}$. Figure $8 \mathrm{c}$ shows the contact resistance as a function of sheet resistivity in order to compare with Fig. 7 . The shaded area corresponds to $\Delta R / R>2 \%$ for $\mathrm{WSe}_{2}$ at $4 \mathrm{~K}$ (note that the corresponding region for $\mathrm{WS}_{2}$ at $74 \mathrm{~K}$ would be very similar). The data for backgated single-layer $\mathrm{WS}_{2}(T=74 \mathrm{~K})^{99}$ lies far above the limit, whereas electrolyte-gated single-layer $\mathrm{WSe}_{2}(T=4 \mathrm{~K})^{100}$ displays a much more appropriate contact resistance range. In addition to making the contact resistance lower, this kind of doping also reduces the width of the depletion region, thus making the contact more suitable for spin injection ${ }^{118}$. Chloride doping ${ }^{95}$ in 5-7 layers of $\mathrm{WS}_{2}$ (square symbol) has also been reported as a representative example of molecular doping ${ }^{94,119-123}$, which is another way of decreasing $R_{\mathrm{C}}$. In Fig. $8 \mathrm{~d}$ we plot the optimal contact resistance needed to achieve maximum magnetoresistance in $\mathrm{WS}_{2}$, along with its evolution as temperature is varied from $73 \mathrm{~K}$ to room temperature. These values were calculated using experimentally determined temperaturedependent figures of the mobility ${ }^{99}$ and spin lifetime ${ }^{124}$. It is clear to see that as the temperature is increased the signal vanishes and the requirement on contact resistance becomes more stringent.

The use of tunnel barriers has proven to be efficient in reducing the contact resistance and the SB between $\mathrm{MoS}_{2}$ and ferromagnetic contacts $^{58,60,61}$. Combined with molecular or electrolyte doping strategies, this could lead to the demonstration of lateral spin valves based on $\mathrm{WS}_{2}$ or $\mathrm{WSe}_{2}$ transistors in the near future.

\section{Summary and outlook}

Realizing good electrical contacts is a prerequisite for harnessing the full potential of 2D SCs. The atomic-scale thickness and pristine surfaces of 2D materials make it difficult to reduce the contact resistance. New theoretical models and experimental approaches better suited to the low-dimensionality of the semiconducting material must be developed. Recent years have shown impressive progress towards solving this problem. Several routes towards high-quality electrical contacts have been identified, the most promising being the realization of seamless electrical contacts, in which native chemical bonds allow much easier charge transport and thereby lower contact resistances. For example, metallic TMDCs can be used as covalently bonded electrical contacts to semiconducting TMDCs, or $s p^{2}$ carbon-carbon covalent bonding could be retained at grapheneGNR junctions. However, most of the results so far have been obtained on graphene and $\mathrm{MoS}_{2}$. Material-specific properties such as constituent elements and atomic defects can strongly influence the electrical properties of the device. In this respect, our understanding of these contacts is still very limited, and more systematic studies are needed, particularly on other TMDCs.

Spintronics, another research area that depends critically on controlling contact resistance, will gain prominence with the rising value of TMDC materials for spintronic and valleytronic applications. Initial estimates show that it should be possible to realize efficient spin injection into this class of materials, giving hope that the first experimental realizations of this will soon be reported.

Received 30 March 2015; accepted 18 September 2015; published online 20 November 2015

\section{References}

1. Iijima, S. Helical microtubules of graphitic carbon. Nature 354, 56-58 (1991).

2. Hayden, O., Agarwal, R. \& Lu, W. Semiconductor nanowire devices. Nano Today 3, 12-22 (2008).

3. Novoselov, K. S. et al. Electric field effect in atomically thin carbon films. Science 306, 666-669 (2004).

4. Radisavljevic, B., Radenovic, A., Brivio, J., Giacometti, V. \& Kis, A. Single-layer $\mathrm{MoS}_{2}$ transistors. Nature Nanotech. 6, 147-150 (2011).

5. Fang, H. et al. High-performance single layered WSe $\mathrm{C}_{2}$-FETs with chemically doped contacts. Nano Lett. 12, 3788-3792 (2012).

6. Liu, W. et al. Role of metal contacts in designing high-performance monolayer n-type WSe ${ }_{2}$ field effect transistors. Nano Lett. 13, 1983-1990 (2013).

7. Sarkar, D. et al. A subthermionic tunnel field-effect transistor with an atomically thin channel. Nature 526, 91-95 (2015).

8. Splendiani, A. et al. Emerging photoluminescence in monolayer $\mathrm{MoS}_{2}$. Nano Lett. 10, 1271-1275 (2010).

9. Mak, K. F., Lee, C., Hone, J., Shan, J. \& Heinz, T. F. Atomically thin $\mathrm{MoS}_{2}$ : A new direct-gap semiconductor. Phys. Rev. Lett. 105, 136805 (2010).

10. Coleman, J. N. et al. Two-dimensional nanosheets produced by liquid exfoliation of layered materials. Science 331, 568-571 (2011).

11. Yoon, Y., Ganapathi, K. \& Salahuddin, S. How good can monolayer $\mathrm{MoS}_{2}$ transistors be? Nano Lett. 11, 3768-3773 (2011).

12. Cao, W., Kang, J., Sarkar, D., Liu, W. \& Banerjee, K. 2D semiconductor FETs-Projections and design for sub-10 nm VLSI. IEEE Trans. Electron Dev. 62, 3459-3469 (2015).

13. Sarkar, D. et al. $\mathrm{MoS}_{2}$ field-effect transistor for next-generation label-free biosensors. ACS Nano 8, 3992-4003 (2014).

14. Lembke, D. \& Kis, A. Breakdown of high-performance monolayer $\mathrm{MoS}_{2}$ transistors. ACS Nano 6, 10070-10075 (2012).

15. He, K., Poole, C., Mak, K. F. \& Shan, J. Experimental demonstration of continuous electronic structure tuning via strain in atomically thin $\mathrm{MoS}_{2}$. Nano Lett. 13, 2931-2936 (2013).

16. Conley, H. J. et al. Bandgap engineering of strained monolayer and bilayer $\mathrm{MoS}_{2}$. Nano Lett. 13, 3626-3630 (2013).

17. Castellanos-Gomez, A. et al. Local strain engineering in atomically thin $\mathrm{MoS}_{2}$. Nano Lett. 13, 5361-5366 (2013). 
18. Bertolazzi, S., Brivio, J. \& Kis, A. Stretching and breaking of ultrathin $\mathrm{MoS}_{2}$. ACS Nano 5, 9703-9709 (2011).

19. Geim, A. K. \& Grigorieva, I. V. Van der Waals heterostructures. Nature 499, 419-425 (2013).

20. Li, L. et al. Black phosphorus field-effect transistors. Nature Nanotech. 9, 372-377 (2014)

21. Tao, L. et al. Silicene field-effect transistors operating at room temperature. Nature Nanotech. 10, 227-231 (2015).

22. Lopez-Sanchez, O., Lembke, D., Kayci, M., Radenovic, A. \& Kis, A Ultrasensitive photodetectors based on monolayer $\mathrm{MoS}_{2}$. Nature Nanotech. 8, 497-501 (2013)

23. Krasnozhon, D., Lembke, D., Nyffeler, C., Leblebici, Y. \& Kis, A $\mathrm{MoS}_{2}$ transistors operating at gigahertz frequencies. Nano Lett. 14, 5905-5911 (2014)

24. Das, S., Chen, H.-Y., Penumatcha, A. V. \& Appenzeller, J. High performance multilayer $\mathrm{MoS}_{2}$ transistors with scandium contacts. Nano Lett. 13, 100-105 (2013).

25. Liu, H., Neal, A. T. \& Ye, P. D. Channel length scaling of $\mathrm{MoS}_{2}$ MOSFETs. ACS Nano 6, 8563-8569 (2012).

26. Liu, H. et al. Statistical study of deep submicron dual-gated field-effect transistors on monolayer chemical vapor deposition molybdenum disulfide films. Nano Lett. 13, 2640-2646 (2013).

27. Landauer, R. Spatial variation of currents and fields due to localized scatterers in metallic conduction. IBM J. Res. Dev. 1, 223-231 (1957).

28. Sharvin, Y. V. A possible method for studying Fermi surfaces. Sov. Phys. JETP 21, 655-656 (1965).

29. Jena, D., Banerjee, K. \& Xing, G. H. 2D crystal semiconductors: Intimate contacts. Nature Mater. 13, 1076-1078 (2014).

30. Song, S. M., Park, J. K., Sul, O. J. \& Cho, B. J. Determination of work function of graphene under a metal electrode and its role in contact resistance. Nano Lett. 12, 3887-3892 (2012).

31. Wang, L. et al. One-dimensional electrical contact to a two-dimensional material. Science 342, 614-617 (2013).

32. Kang, J., Liu, W., Sarkar, D., Jena, D. \& Banerjee, K. Computational study of metal contacts to monolayer transition-metal dichalcogenide semiconductors. Phys. Rev. X 4, 031005 (2014).

33. Matsuda, Y., Deng, W.-Q. \& Goddard, W. A. Contact resistance for 'endcontacted' metal-graphene and metal-nanotube interfaces from quantum mechanics. J. Phys. Chem. C 114, 17845-17850 (2010).

34. Kang, J., Sarkar, D., Liu, W., Jena, D. \& Banerjee, K. A computational study of metal-contacts to beyond-graphene 2D semiconductor materials. IEEE Int. Electron Dev. Meet. 407-410 (2012).

35. Liu, W. et al. High-performance few-layer- $\mathrm{MoS}_{2}$ field-effect-transistor with record low contact-resistance. IEEE Int. Electron Dev. Meet. 19.4.1-19.4.4 (2013)

36. Khatami, Y., Li, H., Xu, C. \& Banerjee, K. Metal-to-multilayer-graphene contact-Part II: analysis of contact resistance. IEEE Trans. Electron. Dev. 59, 2453-2460 (2012)

37. Khatami, Y., Li, H., Xu, C. \& Banerjee, K. Metal-to-multilayer-graphene contact-Part I: contact resistance modeling. IEEE Trans. Electron. Dev. 59, 2444-2452 (2012).

38. Chu, T. \& Chen, Z. Understanding the electrical impact of edge contacts in few-layer graphene. ACS Nano 8, 3584-3589 (2014).

39. Park, H.-Y. et al. Contact resistance reduction on graphene through n-type doping and one-dimensional edge contact. MRS Spring Meeting T14.14 (2015).

40. Stokbro, K., Engelund, M. \& Blom, A. Atomic-scale model for the contact resistance of the nickel-graphene interface. Phys. Rev. B 85, 165442 (2012).

41. Popov, I., Seifert, G. \& Tománek, D. Designing electrical contacts to $\mathrm{MoS}_{2}$ monolayers: a computational study. Phys. Rev. Lett. 108, 156802 (2012).

42. Kang, J., Liu, W. \& Banerjee, K. High-performance $\mathrm{MoS}_{2}$ transistors with lowresistance molybdenum contacts. Appl. Phys. Lett. 104, 093106 (2014).

43. Gan, L.-Y., Zhao, Y.-J., Huang, D. \& Schwingenschlögl, U. First-principles analysis of $\mathrm{MoS}_{2} / \mathrm{Ti}_{2} \mathrm{C}$ and $\mathrm{MoS}_{2} / \mathrm{Ti}_{2} \mathrm{CY}_{2}(\mathrm{Y}=\mathrm{F}$ and $\mathrm{OH})$ all-2D semiconductor/ metal contacts. Phys. Rev. B 87, 245307 (2013).

44. Leong, W. S., Nai, C. T. \& Thong, J. T. L. What does annealing do to metalgraphene contacts? Nano Lett. 14, 3840-3847 (2014)

45. Gong, C., Colombo, L., Wallace, R. M. \& Cho, K. The unusual mechanism

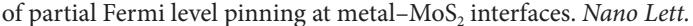
14, 1714-1720 (2014)

46. Ouyang, Y. \& Guo, J. Carbon-based nanomaterials as contacts to graphene nanoribbons. Appl. Phys. Lett. 97, 263115 (2010).

47. Kang, J., Sarkar, D., Khatami, Y. \& Banerjee, K. Proposal for all-graphene monolithic logic circuits. Appl. Phys. Lett. 103, 083113 (2013).

48. Nakanishi, T., Koshino, M. \& Ando, T. Transmission through a boundary between monolayer and bilayer graphene. Phys. Rev. B 82, 125428 (2010).

49. Kappera, R. et al. Phase-engineered low-resistance contacts for ultrathin $\mathrm{MoS}_{2}$ transistors. Nature Mater. 13, 1128-1134 (2014).
50. Kappera, R. et al. Metallic $1 \mathrm{~T}$ phase source/drain electrodes for field effect transistors from chemical vapor deposited $\mathrm{MoS}_{2}$. APL Mater. 2, 092516 (2014)

51. Lin, Y.-C., Dumcenco, D. O., Huang, Y.-S. \& Suenaga, K. Atomic mechanism of the semiconducting-to-metallic phase transition in single-layered $\mathrm{MoS}_{2}$. Nature Nanotech. 9, 391-396 (2014).

52. Sze, S. M. \& Ng, K. K. Physics of Semiconductor Devices (Wiley, 2006).

53. Leonard, F. \& Talin, A. A. Electrical contacts to one- and two-dimensional nanomaterials. Nature Nanotech. 6, 773-783 (2011).

54. Banerjee, K., Amerasekera, A., Dixit, G. \& Hu, C. Temperature and current effects on small-geometry-contact resistance. IEEE Int. Electron Dev. Meet. 115-118 (1997).

55. Heine, V. Theory of surface states. Phys. Rev. 138, A1689-A1696 (1965).

56. Anwar, A., Nabet, B., Culp, J. \& Castro, F. Effects of electron confinement on thermionic emission current in a modulation doped heterostructure. J. Appl. Phys. 85, 2663-2666 (1999).

57. Mott, N. F. Impurity band conduction. Experiment and theory. The metal-insulator transition in an impurity band. J. Phys. Colloq. 37, C4-301-C4-306 (1976).

58. Chen, J.-R. et al. Control of Schottky barriers in single layer $\mathrm{MoS}_{2}$ transistors with ferromagnetic contacts. Nano Lett. 13, 3106-3110 (2013).

59. Qiu, H. et al. Electrical characterization of back-gated bi-layer $\mathrm{MoS}_{2}$ field-effect transistors and the effect of ambient on their performances. Appl. Phys. Lett. 100, 123104 (2012).

60. Dankert, A., Langouche, L., Kamalakar, M. V. \& Dash, S. P. High-performance molybdenum disulfide field-effect transistors with spin tunnel contacts. ACS Nano 8, 476-482 (2014).

61. Wang, W. et al. Controllable Schottky barriers between $\mathrm{MoS}_{2}$ and permalloy. Sci. Rep. 4, 6928 (2014).

62. Guo, Y. et al. Study on the resistance distribution at the contact between molybdenum disulfide and metals. ACS Nano 8, 7771-7779 (2014).

63. Radisavljevic, B. \& Kis, A. Mobility engineering and a metal-insulator transition in monolayer $\mathrm{MoS}_{2}$. Nature Mater. 12, 815-820 (2013).

64. Chuang, H.-J. et al. High mobility WSe $e_{2}$ p- and n-type field-effect transistors contacted by highly doped graphene for low-resistance contacts. Nano Lett. 14, 3594-3601 (2014)

65. Liu, H. et al. Phosphorene: An unexplored 2D semiconductor with a high hole mobility. ACS Nano 8, 4033-4041 (2014).

66. Lince, J., Carré, D. \& Fleischauer, P. Schottky-barrier formation on a covalent semiconductor without Fermi-level pinning: The metal- $\mathrm{MoS}_{2}(0001)$ interface. Phys. Rev. B 36, 1647-1656 (1987).

67. Jaegermann, W., Pettenkofer, C. \& Parkinson, B. Cu and Ag deposition on layered p-type $\mathrm{WSe}_{2}$ : Approaching the Schottky limit. Phys. Rev. B 42, 7487-7496 (1990).

68. Schlaf, R., Klein, A., Pettenkofer, C. \& Jaegermann, W. Laterally inhomogeneous surface-potential distribution and photovoltage at clustered In/WSe ${ }_{2}$ (0001) interfaces. Phys. Rev. B 48, 14242-14252 (1993)

69. Klein, A., Pettenkofer, C., Jaegermann, W., Lux-Steiner, M. \& Bucher, E. A photoemission study of barrier and transport properties of the interfaces of $\mathrm{Au}$ and $\mathrm{Cu}$ with WSe ${ }_{2}(0001)$ surfaces. Surf. Sci. 321, 19-31 (1994).

70. Maurel, C., Coratger, R., Ajustron, F., Beauvillain, J. \& Gerard, P. Electrical characteristics of metal/semiconductor nanocontacts using light emission in a scanning tunneling microscope. J. Appl. Phys. 94, 1979-1982 (2003).

71. Maurel, C., Ajustron, F., Péchou, R., Seine, G. \& Coratger, R. Electrical behavior of the $\mathrm{Au} / \mathrm{MoS}_{2}$ interface studied by light emission induced by scanning tunneling microscopy. Surf. Sci. 600, 442-447 (2006).

72. Dhanker, R., Chopra, N. \& Giebink, N. C. Plasmonic internal photoemission for accurate device in situ measurement of metal-organic semiconductor injection barriers. Adv. Funct. Mater. 24, 4775-4781 (2014).

73. Afanas'ev, V. V. Internal Photoemission Spectroscopy: Fundamentals and Recent Advances (Elsevier, 2014).

74. Gourmelon, E., Bernède, J. C., Pouzet, J. \& Marsillac, S. Textured $\mathrm{MoS}_{2}$ thin films obtained on tungsten: Electrical properties of the W/MoS 2 contact. J. Appl. Phys. 87, 1182-1186 (2000)

75. McDonnell, S., Addou, R., Buie, C., Wallace, R. M. \& Hinkle, C. L. Defect-dominated doping and contact resistance in $\mathrm{MoS}_{2}$. ACS Nano 8, 2880-2888 (2014)

76. Werner, J. H. \& Güttler, H. H. Barrier inhomogeneities at Schottky contacts. J. Appl. Phys. 69, 1522-1533 (1991).

77. Lin, Y.-F. et al. Barrier inhomogeneities at vertically stacked graphene-based heterostructures. Nanoscale 6, 795-799 (2013).

78. Mathai, A. J., Patel, K. D. \& Srivastava, R. Studies on In-pWSe $e_{2}$ Schottky diode by current-voltage-temperature method. Thin Sol. Film. 518, 4417-4424 (2010).

79. McDonnell, S. et al. Hole contacts on transition metal dichalcogenides: Interface chemistry and band alignments. ACS Nano 8, 6265-6272 (2014).

80. Park, W. et al. Contact resistance reduction using Fermi level de-pinning layer for $\mathrm{MoS}_{2}$ FETs. IEEE Int. Electron Dev. Meet. 5.1.1-5.1.4 (2014). 
81. Leong, W. S. et al. Low resistance metal contacts to $\mathrm{MoS}_{2}$ devices with nickel-etched-graphene electrodes. ACS Nano 9, 869-877 (2015).

82. Chuang, S. et al. $\mathrm{MoS}_{2}$ p-type transistors and diodes enabled by high work function $\mathrm{MoO}_{x}$ contacts. Nano Lett. 14, 1337-1342 (2014).

83. Baugher, B. W. H., Churchill, H. O. H., Yang, Y. \& Jarillo-Herrero, P. Optoelectronic devices based on electrically tunable $\mathrm{p}-\mathrm{n}$ diodes in a monolayer dichalcogenide. Nature Nanotech. 9, 262-267 (2014).

84. Cheng, R. et al. Electroluminescence and photocurrent generation from atomically sharp $\mathrm{WSe}_{2} / \mathrm{MoS}_{2}$ heterojunction $\mathrm{p}-\mathrm{n}$ diodes. Nano Lett. 14, 5590-5597 (2014).

85. Lee, C.-H. et al. Atomically thin $\mathrm{p}-\mathrm{n}$ junctions with van der Waals heterointerfaces. Nature Nanotech. 9, 676-681 (2014).

86. Podzorov, V., Gershenson, M. E., Kloc, C., Zeis, R. \& Bucher, E. Highmobility field-effect transistors based on transition metal dichalcogenides. Appl. Phys. Lett. 84, 3301-3303 (2004).

87. Cohen, S. S. Contact resistance and methods for its determination. Thin Sol. Film. 104, 361-379 (1983).

88. Berger, H. H. Models for contacts to planar devices. Solid State Electron. 15, 145-158 (1972)

89. Scott, D. B., Hunter, W. R. \& Shichijo, H. A transmission line model for silicided diffusions: Impact on the performance of VLSI circuits. IEEE J. Solid-State Circuits 17, 281-291 (1982).

90. Nagashio, K., Nishimura, T., Kita, K. \& Toriumi, A. Metal/graphene contact as a performance killer of ultra-high mobility graphene analysis of intrinsic mobility and contact resistance. IEEE Int. Electron Dev. Meet. 1-4 (2009).

91. Xia, F., Perebeinos, V., Lin, Y., Wu, Y. \& Avouris, P. The origins and limits of metal-graphene junction resistance. Nature Nanotech. 6, 179-184 (2011).

92. Liu, H. et al. Switching mechanism in single-layer molybdenum disulfide transistors: An insight into current flow across Schottky barriers. ACS Nano 8, 1031-1038 (2014).

93. Baugher, B. W. H., Churchill, H. O. H., Yang, Y. \& Jarillo-Herrero, P. Intrinsic electronic transport properties of high-quality monolayer and bilayer $\mathrm{MoS}_{2}$. Nano Lett. 13, 4212-4216 (2013).

94. Kiriya, D., Tosun, M., Zhao, P., Kang, J. S. \& Javey, A. Air-stable surface charge transfer doping of $\mathrm{MoS}_{2}$ by benzyl viologen. J. Am. Chem. Soc. 136, 7853-7856 (2014).

95. Yang, L. et al. Chloride molecular doping technique on $2 \mathrm{D}$ materials: $\mathrm{WS}_{2}$ and $\mathrm{MoS}_{2}$. Nano Lett. 14, 6275-6280 (2014).

96. Cui, X. et al. Multi-terminal transport measurements of $\mathrm{MoS}_{2}$ using a van der Waals heterostructure device platform. Nature Nanotech. 10, 534-540 (2015).

97. Li, S.-L. et al. Thickness scaling effect on interfacial barrier and electrical contact to two-dimensional $\mathrm{MoS}_{2}$ layers. ACS Nano 8, 12836-12842 (2014).

98. Liu, W., Sarkar, D., Kang, J., Cao, W. \& Banerjee, K. Impact of contact on the operation and performance of back-gated monolayer $\mathrm{MoS}_{2}$ field-effect-transistors. ACS Nano 9, 7904-7912 (2015).

99. Ovchinnikov, D., Allain, A., Huang, Y.-S., Dumcenco, D. \& Kis, A. Electrical transport properties of single-layer $\mathrm{WS}_{2}$. ACS Nano 8, 8174-8181 (2014).

100. Allain, A. \& Kis, A. Electron and hole mobilities in single-layer $\mathrm{WSe}_{2}$. ACS Nano 8, 7180-7185 (2014)

101. Yoon, J. et al. Highly flexible and transparent multilayer $\mathrm{MoS}_{2}$ transistors with graphene electrodes. Small 9, 3295-3300 (2013).

102. Bertolazzi, S., Krasnozhon, D. \& Kis, A. Nonvolatile memory cells based on $\mathrm{MoS}_{2}$ /graphene heterostructures. ACS Nano 7, 3246-3252 (2013).

103. Yu, L. et al. Graphene/ $\mathrm{MoS}_{2}$ hybrid technology for large-scale twodimensional electronics. Nano Lett. 14, 3055-3063 (2014).

104. Ma, Y., Dai, Y., Guo, M., Niu, C. \& Huang, B. Graphene adhesion on $\mathrm{MoS}_{2}$ monolayer: An ab initio study. Nanoscale 3, 3883-3887 (2011).

105. Xu, S. et al. High-quality $\mathrm{BN} / \mathrm{WSe} \mathrm{S}_{2} / \mathrm{BN}$ heterostructure and its quantum oscillations. Preprint at http://arxiv.org/abs/1503.08427 (2015).

106. Xiao, D., Liu, G.-B., Feng, W., Xu, X. \& Yao, W. Coupled spin and valley physics in monolayers of $\mathrm{MoS}_{2}$ and other group-VI dichalcogenides. Phys. Rev. Lett. 108, 196802 (2012).

107. Gong, K. et al. Electric control of spin in monolayer WSe $\mathrm{W}_{2}$ field effect transistors. Nanotechnology 25, 435201 (2014).
108. Mak, K. F., McGill, K. L., Park, J. \& McEuen, P. L. The valley Hall effect in $\mathrm{MoS}_{2}$ transistors. Science 344, 1489-1492 (2014).

109. Dolui, K., Narayan, A., Rungger, I. \& Sanvito, S. Efficient spin injection and giant magnetoresistance in $\mathrm{Fe} / \mathrm{MoS}_{2} / \mathrm{Fe}$ junctions. Phys. Rev. B 90, 041401 (2014)

110. Baibich, M. N. et al. Giant magnetoresistance of (001)Fe/(001)Cr magnetic superlattices. Phys. Rev. Lett. 61, 2472-2475 (1988).

111. Schmidt, G., Ferrand, D., Molenkamp, L. W., Filip, A. T. \& van Wees, B. J. Fundamental obstacle for electrical spin injection from a ferromagnetic metal into a diffusive semiconductor. Phys. Rev. B 62, R4790-R4793 (2000).

112. Rashba, E. I. Theory of electrical spin injection: Tunnel contacts as a solution of the conductivity mismatch problem. Phys. Rev. B 62, R16267-R16270 (2000).

113. Fert, A. \& Jaffrès, H. Conditions for efficient spin injection from a ferromagnetic metal into a semiconductor. Phys. Rev. B 64, 184420 (2001).

114. Takahashi, S. \& Maekawa, S. Spin injection and detection in magnetic nanostructures. Phys. Rev. B 67, 052409 (2003).

115. Han, W. et al. Tunneling spin injection into single layer graphene. Phys. Rev. Lett. 105, 167202 (2010)

116. Tombros, N., Jozsa, C., Popinciuc, M., Jonkman, H. T. \& van Wees, B. J. Electronic spin transport and spin precession in single graphene layers at room temperature. Nature 448, 571-574 (2007).

117. Seneor, P. et al. Spintronics with graphene. MRS Bull. 37, 1245-1254 (2012).

118. Albrecht, J. D. \& Smith, D. L. Electron spin injection at a Schottky contact. Phys. Rev. B 66, 113303 (2002).

119. Zhao, P. et al. Air stable p-doping of $\mathrm{WSe}_{2}$ by covalent functionalization. ACS Nano 8, 10808-10814 (2014).

120. Chen, K. et al. Air stable n-doping of WSe ${ }_{2}$ by silicon nitride thin films with tunable fixed charge density. APL Mater. 2, 092504 (2014).

121. Fang, H. et al. Degenerate n-doping of few-layer transition metal dichalcogenides by potassium. Nano Lett. 13, 1991-1995 (2013).

122. Farmer, D. B. et al. Chemical doping and electron-hole conduction asymmetry in graphene devices. Nano Lett. 9, 388-392 (2009).

123. Sarkar, D. et al. Functionalization of transition metal dichalcogenides with metallic nanoparticles: Implications for doping and gas-sensing. Nano Lett. 15, 2852-2862 (2015)

124. Mai, C. et al. Exciton valley relaxation in a single layer of $\mathrm{WS}_{2}$ measured by ultrafast spectroscopy. Phys. Rev. B 90, 041414 (2014).

125. Mai, C. et al. Many-body effects in valleytronics: Direct measurement of valley lifetimes in single-layer $\mathrm{MoS}_{2}$. Nano Lett. 14, 202-206 (2014).

126. Kumar, N., He, J., He, D., Wang, Y. \& Zhao, H. Valley and spin dynamics in $\mathrm{MoSe}_{2}$ two-dimensional crystals. Nanoscale 6, 12690-12695 (2014).

127. Wang, G. et al. Valley dynamics probed through charged and neutral exciton emission in monolayer WSe $\mathrm{W}_{2}$. Phys. Rev. B 90, 075413 (2014).

128. Du, Y., Liu, H., Deng, Y. \& Ye, P. D. Device perspective for black phosphorus field-effect transistors: Contact resistance, ambipolar behavior, and scaling. ACS Nano 8, 10035-10042 (2014).

\section{Acknowledgements}

This work was performed in frames of the Marie Curie ITN network MoWSeS (grant no. 317451). We acknowledge funding by the European Commission under the Graphene Flagship (grant agreement no. 604391), the US National Science Foundation under Grant CCF-1162633 and the US Air Force Office of Scientific Research under Grant FA9550-14-1-0268 (R18641).

\section{Additional information}

Reprints and permissions information is available online at www.nature.com/reprints. Correspondence should be addressed to K.B. and A.K.

\section{Competing financial interests}

The authors declare no competing financial interests. 